PPPL --2801

DE92 003507

\title{
Effect of Nonaxisymmetric Perturbations on the Structure of a Tokamak Poloidal Divertor
}

\author{
N. Pomphrey and A. Reiman \\ Princeton Plasma Physics Laboratory, Princeton University \\ Princeton, N. J. 08543
}

\begin{abstract}
The effects of $n=1$ nonaxisymmetric perturbations on a tokamak poloidal divertor are described. Despite the existence of a region of chaotic field line trajectories outside the last closed flux surface, the footprint of the trajectories on the divertor plates is found to be largely coherent, forming a spiral structure. At a fixed toroidal angle, the footprint exhibits a bifurcation similar to that seen experimentally on DIII-D [Nucl. Fusion 28(5),902 (1988)]. For field errors of the magnitude that exist in present day tokamaks, the width of the nonaxisymmetric structure in the divertor footprint is comparable to the width of the scrape-off layer.
\end{abstract}




\section{Introduction}

Poloidal divertors are a common feature of large present day tokamak devices, and are proposed in the $\mathrm{BPX}^{1}$ and $\mathrm{ITER}^{2}$ designs. Conventional modeling of divertor effects assumes axisymmetry. ${ }^{3}$ In this paper we study the effects of small nonaxisymmetric perturbations on the structure of a simple divertor. We find that perturbation amplitudes typical of error fields in existing and contemplated tokamaks produce large nonaxisymmetries that should be taken into account in realistic divertor modeling. We will see that the nonaxisymmetry shows both chaotic and coherent features.

We are ultimately interested in issues related to heat flux deposition at the divertor plates. Since particle motion is predominantly parallel to the magnetic field lines, it is clear that the magnetic field line trajectories are a strong determinant of the heat deposition. In this paper, we concentrate on characterizing these trajectories. In particular, we will be especially interested in the footprint of the field lines on the divertor plates.

The structure of tokamak magnetic field lines in the presence of an axisymmetric poloidal divertor is shown in Fig. 1. (The figure shows a double null divertor, that is. a divertor with two $X$-lines.) Inside the separatrix, field lines lie on nested flux surfaces. Just outside the separatrix, the field lines are open and intersect the divertor plates. Particles can move rapidly along the field lines to the divertor plates, implying that the plasma density and energy fall off rapidly as a function of distance from the separatrix. The scale lengths over which the density and energy go to zero define the density and energy scrape-off widths. For BPX parameters ${ }^{1}\left(R_{0}=2.59 m, a=0.80 m\right)$, for example, the energy scrape-off width is predicted to be about $0.4 \mathrm{~cm}$ at the midplane.

It is well known that a symmetry breaking perturbation in a dynamical system produces a stochastic layer at a separatrix. ${ }^{4}$ For tokamaks, magnetic field errors due to imperfect construction of the poloidal and toroidal field coils provide this symmetry breaking perturbation. This suggests an investigation of the effect of field errors on the physics of the divertor region of tokamaks.

In bounded Hamiltonian systems, one deals with a situation where the orbits both inside and outside the separatrix are quasiperiodic. For tokamaks this would correspond to the situation where there is a diverted plasma with 
no divertor plates or material surfaces to limit the magnetic field lines in the vacuum. A nonaxisymmetric perturbation produces a stochastic layer about the unperturbed separatrix. Magnetic field lines in this layer wander throughout a volume, rather than lying on a surface, and have infinite length. The orbits are bounded by flux surfaces on either side of the stochastic layer and have an infinite number of encounters with the $X$ points. Coherent nonaxisymmetries are associated with the shape of the boundary of the stochastic layer, and with remnant islands within the stochastic layer.

Consider the effect of introducing divertor plates to this picture. All field line trajectories with initial conditions just outside the last closed flux surface of the plasma region now terminate at the divertor plates after a finite number of encounters with the $X$ points. Since these trajectories have finite length they are no longer stochastic in the usual time-asymptotic sense. One must ask whether the nonaxisymmetric perturbation significantly affects a trajectory over its finite length. The investigation leads us to relate the problem of trajectory evolution in the presence of divertor plates to the general field of chaotic scattering, ${ }^{5}$ investigated in the context of plasmas by Lau and Finn, ${ }^{6}$ who studied plasmoid models relevant to the solar corona.

It is natural to address the question of divertor nonaxisymmetry in two steps. The first step is to investigate divertor nonaxisymmetry in the absence of divertor plates. If the width associated with the nonaxisymmetry were small for perturbation amplitudes of interest, then it is clear that the additional complication introduced by the divertor plates would be irrelevant. We investigate the field line trajectories in the absence of divertor plates in Sec. III. We find that the width associated with the nonaxisymmetries is large compared to the scrape-off layer (SOL) width for typical error field amplitudes.

The second step is to investigate the field line trajectories in the presence of divertor plates. We do that in Sec. IV.

The effect of magnetic perturbations on a divertor separatrix has previously been studied by Tomita, Seki, and .Momota, ${ }^{7}$ and by Boozer and Rechester. $^{8}$ These papers employ the Chirikov overlap criterion to estimate the width of the stochastic layer in the absence of divertor plates. Both papers focus on the nonaxisymmetric perturbation due to magnetic field ripple. The effect due to this is found to be small because of an exponential dependence on the toroidal mode number of the perturbation. Boozer and 
Rechester also offer a rough estimate of the stochastic layer width for a more general perturbation of their single null divertor model in the absence of divertor plates. The predictions of this formula are roughly consistent with the numerical resulis of Sec. III for stochastic layer widths in double null divertors without divertor plates. The key element not considered in this early work is the effect of the divertor plates in terminating field line trajectories.

LaHaye $^{9}$ has investigated some details of field line trajectories in the divertor region of the DIII-D tokamak. His field line tracing is for full toroidal geometry and uses information from experimental measurements of the poloidal field irregularities in DIII-D to define the dominant component of the error field spectrum. The results clearly show that error fields have a significant effect on the details of trajectory evolution even when the finite length of the trajectories is taken into account.

In this paper, we choose a simple model of a tokamak divertor defined by three straight wires with co-directional currents to which an $n=1$ field error perturbation is added whose magnitude is typical of field errors that exist in present day tokamaks. The simple geometry allows us to follow thousands of field line trajectories, and to construct detailed magnetic footprints of the field line intersections with the divertor plates. The footprints are found to have a generic spiral structure, very suggestive of the observed poloidal bifurcation in the heat deposition profiles in DIII-D. ${ }^{10}$

\section{The Divertor Model}

A simple model of an axisymmetric divertor region is seen in Fig. 1. The system comprises three straight wires with co-directional currents. One of the wires (representing the plasma), is placed at $x=0, z=0$ and carries a current of $+I_{p}$ in the $\phi$ direction. The other two wires (coils), are located at $x=0, z= \pm z_{c}$, and each carries a current of $+I_{c}$.

The magnetic field for this unperturbed configuration can be written as

$$
\mathrm{B}=\hat{\phi} \times \nabla \Psi_{0}+B_{0} \hat{\phi},
$$

where

$$
\Psi_{0}=\mu_{0} I_{p} / 4 \pi \log \left(r_{+}^{2 \gamma} r^{2} r_{-}^{2 \gamma}\right)
$$


and

$$
\gamma \equiv I_{c} /{ }^{r} p
$$

For any positive value of $\gamma$, a separatrix surface divides the plasma region from the coils. Denoting the midplane radius of the separatrix by $a$, the separatrix X-point height by $z_{N}$, and the separatrix elongation by $\kappa_{N}=z_{N} / a$, the relationship between the separatrix geometry and the wire positions and currents is given by

$$
\begin{gathered}
\kappa_{N}^{2}=\left(1+\left(1+\kappa_{N}^{-2}\right) /(2 \gamma)\right)^{2 \gamma}, \\
z_{N}= \pm z_{c} /(2 \gamma+1)^{1 / 2} .
\end{gathered}
$$

Figure 2 shows that $\kappa_{N}$ is a monotonic function of $\gamma$ with asymptotic maximum at $\kappa_{N}^{\max } \approx 1.87$. In order to make the model divertor geometry relevant to open divertor configurations such as the proposed BPX experiment, it is necessary to avoid large values of $\gamma$. This limits the attainable separatrix elongation of the model. For the numerical calculations shown in this paper, we therefore choose $a=0.80$, and $\kappa_{N}=1.60$, giving $\gamma=0.64$, and $z_{c}=1.93$.

It remains to fix the length scale

$$
L=\mu_{0} I_{p} / 4 \pi B_{0}
$$

which is easily identified as the inverse of the safety factor per unit length. For the computations, we choose $L=0.06$ to fix $q_{95}=3.2$, where the subscript on $q$ refers to its evaluation on a flux surface passing through the midplane value $x / a=0.95$. Figure 3 shows a plot of the safety factor in the neighborhood of the separatrix. A logarithmic increase of $q$ is seen as $|x / a-1| \rightarrow 0^{ \pm}$. Since $L$ is positive, magnetic field lines corresponding to $x / a>1$ at $z=0$ rotate in a clockwise poloidal direction around all three wires. For $x / a \gg 1$, the safety factor is determined as if the system were a single wire with current equal to $I_{p}+2 I_{c}$. Thus, $q \sim(x / a)^{2}$ as $x / a \rightarrow \infty$, and a minimum value of $q \approx 3.5$ is obtained for $x / a \approx 1.12$.

To study the effects of non-axisymmetry on the structure of the poloidal divertor, we add an $n=1$ vacuum field perturbation to Eq. (1). The curl and divergence free form we choose is

$$
\delta \mathbf{B}=B_{0} \nabla V,
$$


where

$$
V=\epsilon \frac{R_{0}}{B_{0}} \exp \left(x / R_{0}\right) \cos (\phi)
$$

and where the periodicity length for the "toroidal" angle $\phi$ is chosen to give an "aspect ratio" $a / R_{0}=0.30$, namely $R_{0}=2.67$.

The field line equations resulting from Eqs. (1), (7), and (8) are

$$
\begin{aligned}
& \frac{d x}{d \phi}=\frac{B^{x}}{B^{\phi}} \\
& \frac{d x}{d \phi}=\frac{B^{z}}{B^{\phi}},
\end{aligned}
$$

where

$$
\begin{gathered}
B^{x}=\frac{\partial \Psi_{0}}{\partial z}+\epsilon \exp \left(x / R_{0}\right) \cos (\phi) \\
B^{\phi}=\frac{1}{R_{0}}\left[B_{0}-\epsilon \exp \left(x / R_{0}\right) \sin (\phi)\right]
\end{gathered}
$$

and

$$
B^{z}=-\frac{\partial \Psi_{0}}{\partial x}
$$

These equations are integrated for given initial conditions, $x=x_{0}, \phi=\phi_{0}$, $z=z_{0}$, and selected values of the field perturbation amplitude, $\epsilon / B_{0}$.

Nonaxisymmetric error fields are produced in present day tokamaks by such things as finite tolerances in the installation of the poloidal and toroidal field coils, the presence of bus bars, etc. Typical magnitudes of such fields are $\delta B / B \approx 10^{-4}$ for the low order Fourier components. In JET, ${ }^{11}$ the $m=2$, $n=1$ fourier component of the field error is believed to be of order 1 Gauss at the $q=2$ surface, and to be caused by the toroidal bus bars. In ASDEX, ${ }^{12}$ the $(2,1)$ Fourier component of the error field is believed to be about one or two Gauss, and to be caused by the finite tolerances in the installation of the toroidal field coils. In DIII-D, the field error is believed to be about two Gauss, and to be due to the finite tolerances in the installation of the poloidal field (PF) coils. ${ }^{13}$ The BPX Physics Project ${ }^{1}$ has adopted a tentative field error tolerance of the form

$$
\sum_{1 \leq m / n \leq 5} \frac{1}{n}\left(\frac{m B_{r m n}}{B_{0}}\right)^{1 / 2}<E \approx 0.05 .
$$


The left hand side of this expression is motivated by a rough estimate of the total width of the plasma region occupied by islands, assuming a constant rotational transform. The numerical value of the right hand side comes from extensive field line tracing and three dimensional equilibrium calculations using experimental data. ${ }^{13,1}$ For easy comparison with different devices, the Fourier decompositions used in evaluating the BPX criterion, Eq. (11), are performed on a reference circular surface centered at the major radius $R_{0}$, and having minor radius $r=a$.

In addition to the field error, there is also a nonaxisymmetric component of the magnetic field produced by the discreteness of the toroidal field coils. The magnitude of this "field ripple" is typically much larger than the magnitude of the field error, but it has a high toroidal mode number. The effect of the nonaxisymmetric Fourier components can be expected to decrease exponentially as a function of the toroidal mode number, ${ }^{8,7}$ so that field errors can be expected to have a greater effect on the divertor separatrix.

The scaling with toroidal mode number seen in Eq. (11) motivates the focus in this paper on $n=1$ perturbations. The field error produced by shifts and tilts of the PF coils can be expected to be predominantly $n=1$. The field errors produced by toroidal field coils and buss bars will generally have a broad Fourier spectrum in the toroidal mode number. Because the effects of the $n=1$ Fourier components are expected to dominate, it is reasonable to study the effects of a pure $n=1$ nonaxisymmetric field. The exponential dependence on $x$ in Eq. (8) models the toroidal fall off of a tokamak vacuum field, with the scale length appropriately determined by the aspect ratio.

Equation 11 can be used to estimate a physically relevant value for the tokamak error field magnitude $\epsilon$ of the model perturbation used in our study. For this purpose we use the expression ${ }^{14}$

$$
\exp \left(\frac{a}{R_{0}} \cos \theta\right)=\mathrm{I}_{0}\left(\frac{a}{R_{0}}\right)+\sum_{j=1}^{\infty} 2 \mathrm{I}_{j}\left(\frac{a}{R_{0}}\right) \cos (j \theta),
$$

where $I_{j}$ denotes the Modified Bessel Function of order $j$. Evaluation of the $m=1, n=1$ Fourier component of $\delta B_{x} / B_{0}$ yields $.52 \epsilon / B_{0}$, while the $m=2$, $n=1$ and $m=3, r_{\iota}=1$ Fourier components are $.076 \epsilon / B_{0}$ and $.0057 \epsilon / B_{0}$ respectively. In terms of the BPX field error tolerance, $E=0.05$ would require $\epsilon / B_{0}<1.6 \times 10^{-3}$, with $\delta B_{r 21} / B_{0}<1.2 \times 10^{-4}$. 


\section{Nonaxisymmetric Field Line Trajectories in the Absence of Divertor Plates}

It is convenient to begin our presentation of results by considering field line evolution in the absence of divertor plates. For the three-wire system described in Sec. II, this corresponds to a conventional treatment of the magnetic system as a $1 \frac{1}{2}$ degree of freedom nonintegrable Hamiltonian. Each nonperiodic field line is then an orbit of infinite length in a bounded phase space. For initial conditions close to the separatrix, each orbit has an infinite number of close encounters with each X-point. It is well known that under the action of a nonintegrable perturbation, the magnetic separatrix and nearby surfaces are destroyed, and a stochastic layer develops. The width of the stochastic layer depends on the amplitude of the perturbation.

Results of integrating the field line equations (Eq. 9) for selected initial conditions and perturbation amplitude $\epsilon / B_{0}=1.0 \times 10^{-3}$ are shown in Fig. 4. Each field line is followed for 5000 toroidal circuits, and a Poincaré section is constructed by plotting a point every time the orbit passes through a chosen $\phi$ section. Two sections, corresponding to different values of $\phi$, are shown in the figure. Each section shows two regions of "good" flux surfaces, separated by a stochastic layer. The layer is clearly associated with the region of the unperturbed separatrix. In the inner (plasma) region islands associated with the $q=1$ and $q=3$ orbits are shown. The good surfaces are lirited to $q$ values less than about 3.7. The dashed line in the figure is drawn at constant $\left|z_{p}\right|=1.40$, and we should imagine this to be a possible location for a divertor plate. The plate is separated from the unperturbed $\mathrm{X}$-point by approximately 0.17 units of distance along the direction of the unperturbed separatrix. Within the stochastic layer are island structures, dominated by the $q=5$ islands. Comparing the two section views of the divertor region shows that the $q=5$ island region overlaps the plate to a substantially different extent depending on the $\phi$ value, indicating both a poloidal and a toroidal magnetic asymmetry on the proposed divertor plate surface. Outside of the stochastic layer are good surfaces representing the vacuum region.

The magnetic structure in the divertor region is displayed more clearly in Fig. 5 and Fig. 6, which show, respectively, a magnified view of the lower divertor region outlined by the solid box in Fig. 4, and the section $x$ vs. 
$\phi$ at $z_{p}=-1.40$. To obtain these figures, initial conditions were selected for the orbit integrations at $\phi_{0}=0.00, z_{0}=1.28$, with thirty values of $x_{0}$ chosen at equal intervals between 0.00 , and 0.30 . [Recall that the X-point lies at $(x, z, \phi)=(0.0,1.28,0.0)$.] Each field line was again followed for 5000 toroidal transits. Aided by the natural flux expansion in the divertor region, the richness of the field line structure is readily apparent in these plots. To measure the width of the stochastic region we use the nominal BPX scrapeoff distance as a ruler. At the midplane this value is $\delta x_{S O L}=0.004$. Field line tracing from the midplane yields $\Delta x_{S O L}=0.018$ for the scrapeoff width at the divertor plates. The measured width of the stochastic region at the divertor plates is therefore seen to be an order of magnitude greater than the width of the unperturbed divertor SOL.

Field line following for several values of the perturbation strength shows that the stochastic width at $z_{p}$ scales linearly with $\epsilon / B_{0}$. Furthermore, the stochastic width at the midplane and the stochastic width at the plate are related by the unperturbed flux expansion rule, $\Delta x=4.3 \delta x$. Thus, in the absence of any field line terminating divertor plates, the width of the stochastic layer at the midplane and at the divertor plates for nonaxisymmetric field perturbations typical of present day tokamaks is an order of magnitude greater than the nominal axisymmetric scrapeoff widths.

\section{Field Line Trajectories in the Presence of Diver- tor Plates}

From the point of view of heat flux deposition, the magnetic field lines which connect the plasma region to the divertor plates serve as the conduit for particle exhaust. Since the close tie between particle orbits and field line trajectories is broken once an orbit encounters a divertor plate it follows that a physically relevant field line integration study should terminate the field lines immediately upon first intersecting a plate. This section describes such a study.

To obtain some understanding of the effects of terminating the trajectories at the divertor plates, we perform the following preliminary numerical experiment. For each of several initial values of $x / a \approx 1$ on the midplane, the field line equations are integrated for 20 equally spaced initial values of the toroidal angle, and the number of toroidal transits made by each trajec- 
tory before it intersects the plate is recorded. Results are shown in Fig. 7. The vertical axis denotes initial midplane radius, the horizontal axis denotes initial toroidal angle, and each number in the array of data represents the number of toroidal transits, $N_{\phi}$. Trajectories which fail to intersect a plate within 5000 transits are terminated. These are identified in the figure by the symbol " $\mathbf{X}$ ". The dividing line between initial conditions which lead to trajectories that intersect the plates and initial conditions of trajectories that remain trapped for 5000 transits is considered to represent the approximate edge of the plasma region. The plasma edge in the presence of divertor plates must of course be the same as the plasma edge in the absence cf divertor plates. The $n=1$ modulation of the edge is approximately captured by the function

$$
\left(x_{0} / a\right)_{I N}=0.990+0.004 \sin \left(\phi_{0}\right)
$$

which is represented in Fig. 7 by the lower of the two solid curves.

Outside of the plasma region is a band of $N_{\phi}$ values greater than zero. Much of the band appears to show little correlation between adjacent values of $N_{\phi}$. We will refer to the band as the stochastic layer in the presence of a plate, and justify the reference to stochasticity shortly. Within the band is an island of $\mathbf{X}$ values. By constructing a surface of section for orbits with initial conditions in this region, it is identified as a $q=4$ island that never intersects the divertor plate.

A guide to the location of the outer boundary of the stochastic layer is provided by a second curve in Fig. 7, roughly corresponding to the contour value $N_{\phi}=8$. The reason for choosing this contour value will also be justified shortly. The outer boundary is seen to have an $n=1$ oscillation about $x_{0} / a \approx 1.0$, the location of the unperturbed separatrix. The stochastic layer is therefore predominantly associated with a region inside the unperturbed separatrix. The width of the stochastic layer is $\delta x / a \approx 0.008$, substantially less than the stochastic width in the absence of plates (calculated in the last section to be $\delta x / a \approx 0.07)$, but comparable to the nominal scrapeoff width of $\delta x_{S O L} / a=0.005$.

Since we are interested in the heat deposition on the divertor plates, it is natural to ask what form the footprint of field line intersections with the divertor plate takes for trajectories launched at the edge of the plasma region. The true edge has a complicated structure which would require a significant computational effort to map out. To avoid expending this effort we consider 
a modification of the field line equations which replaces Eq. 9b with

$$
\frac{d z}{d \phi}=\frac{B^{z}}{B^{\phi}}+\eta \frac{z}{R_{0}},
$$

where $\eta$ is a random number whose value changes with each new initial condition. The random number $\eta$ is uniformly distributed in a range between $\eta_{0}$ and $2 \eta_{0}$, where $\eta_{0}$ is a small positive constant which remains fixed for all initial conditions. The effect of the modification on the fleld lines is to introduce a tight spiral in the $x-z$ plane, so that an orbit which initially lies within the plasma region is guaranteed to eventually cross the last closed flux surface and escape to the divertor plate. By choosing random $\eta$ values but the same initial $x, z$ and $\phi$ values, the trajectories cross the true edge of the plasma region at random poloidal and toroidal locations. If the constant $\eta_{0}$ is small enough, the magnetic footprint at the plate can be made indistinguishable from the footprint produced by initial conditions at the true plasma edge.

A suitable value of $\eta_{0}$ was determined from a sequence of numerical experiments on the unperturbed 3-wire system where 2000 trajectories were followed, each with a random $\eta$ value and initial $x / a=0.95, z=0, \phi=0$. The value of $\eta_{0}$ was decreased until all the orbits in a given experiment intersect the plates at the known separatrix location $x_{p}=0.110$ to within five parts in $10^{4}$. This determined $\eta_{0}=1 \times 10^{-5}$. With this value of $\eta_{0}$, the intersections of the orbits with the plate are distributed uniformly in $\phi$.

Having determined $\eta_{0}$ in this way we return to the perturbed system with $\epsilon / B_{0}=1 \times 10^{-3}$, select 2000 initial conditions with random $\eta$ at $x / a=0.985$, $z=0$ and $\phi=0$ and follow each trajectory until it intersects a plate. Figure 7 shows that the chosen initial conditions lie well inside the plasma region. Polar plots of the magnetic footprint of field line intersections with the upper and lower divertor plates are shown in Fig. 8. Since the width of the footprints are small compared with their radial location, it helps to use the quantity $r_{p}=x_{p}-0.10$ as the radial variable for the polar plots. This allows us to choose a radial scale that magnifies the footprints and emphasizes their structure. For reference, the location of the intersection of the unperturbed separatrix (at $x_{p}=0.110$ ) is shown as a dashed circle. The footprint is seen to take the form of a spiral loop structure accompanied by some stochastic spreading. The radial extent of the footprint on the lower plate is $\Delta x_{p} \approx 0.015$, approximately a factor of three wider than the region 
on the upper plate. The disparity of widths is due to the asymmetry of the field perturbation with respect to $x$ [see Eq. (7)] and will be discussed later.

To understand what causes the spiral structure at the plate we return to a consideration of the original field line equations (i.e., $\eta_{0}=0$ ) and perform a series of computations where we follow trajectories with initial conditions on midplane curves parallel to the approximate plasma edge defined by Eq. (12). Specifically, we investigate the footprint of groups of 2000 traiectories equally spaced in initial toroidal angle $\varphi_{0}$, which have initial $x$ values lying on midplane curves defined by

$$
x_{0} / a=\left(x_{0} / a\right)_{I N}+\delta x_{0} / a,
$$

and study the form of the footprint for different values of $\delta x_{0} / a$.

Figures 9a,b, and Fig. 10a display polar plots of the frotprints on the lower divertor plate for curves of initial conditions with $\delta x_{0} / a=0.0140$, 0.0125 , and 0.0120 , respectively. A sharp spiral pattern is seen to develop for this sequence of decreasing values of $\delta x_{0} / a$. To understand why this pattern develops, imagine recasting the original field line equations with the variable $z$ as the dependent variable instead of $\phi$, and consider the evolution of a curve of initial conditions as $z$ is advanced from $z=0$ to the $z$ value of the plate. If the curve, $\mathcal{C}$, lies entirely within a single flux surface then each trajectory shares the same rotational transform. Consequently the change in toroidal angle, $\Delta \phi$, per unit change in $z$ is the same for each trajectory, and the shape of $\mathcal{C}$ is preserved. As an example, consider the unperturbed 3 -wire system and a curve of initial conditions parallel to the unperturbed separatrix. Viewed with the $(x, \phi)$ polar representation, the curve of initial conditions at $z=0$ is a circle. The magnetic footprint on the divertor plate is therefore a circle.

Now consider the case when $\mathcal{C}$ does not lie on a single flux surface. Then the shape of $\mathcal{C}$ must change as $z$ advances since each point of the curve has a different pitch and must move a different $\Delta \phi$ per unit $\Delta z$. The points of $\mathcal{C}$ with the smallest transform have the smallest $\Delta \phi / \Delta z$ and, therefore; move the smallest angular distance. This leads to the development of a spiral pattern as $z$ increases, with an inflection point corresponding to the minimum transform. (A simple model that illustrates the development of the spiral pattern is presented in the Appendix of this paper). The sequence of initial curves leading to Fig. 9 and Fig. 10 have decreasing transform since 
these curves are approaching the plasma edge. 'Therefore, since each plot has advanced the same $z$ with respect to the starting value, the spiral is progressively more developed. Following the notation of Berry et al., ${ }^{15}$ who studied the changing form of curves under the influence of a nonlinear map, we will call the spiral footprint a whorl.

The initial curves with $\delta x_{0} / a=0.0140$ and 0.0125 are sufficiently far from the plasma edge, and the rotational transforms are sufficiently high, that every trajectory intersects the lower divertor plate with $N_{\phi} \leq 1$. Figure 10c shows the dependence of $N_{\phi}$ on the initial angle $\phi_{0}$ for the curve with $\delta x_{0} / a=0.0120$. Most of the orbits reach the lower divertor plate within a single toroidal transit and lead to the sharp spiral pattern shown in Fig. 10a. However there exists a small region of initial conditions near $\phi_{0}=0.4$ where $N_{\phi} \gg 1$. Some of these longer orbits intersect the upper divertor plate producing the footprint shown in Fig. 10b. The remainder intersect the lower divertor plate at the points which do not lie on the sharp spiral.

Figures 11 , and 12 show results for $\delta x_{0} / a=0.007$ and 0.002 , respectively. The curves of initial conditions for these runs lie near the outer and inner edges of the stochastic layer defined by the solid curves in Fig. 7. The magnetic footprints are now seen to be remarkably similar to the footprint in Fig. 8 despite the completely different method used to generate the initial conditions. The footprint is a spiral dressed with a stochastic spread which we will call a fuzzy whorl. Figures $11 \mathrm{c}$ and $12 \mathrm{c}$ show the dependence of $N_{\phi}$ on $\phi_{0}$. As previously noted in regard to Fig. 7, there is little correlation between the field line connection lengths (measured by $N_{\phi}$ ) for nearby initial conditions. The "average" connection length is seen to be greater for the initial curve that lies closest to the plasma edge, as expected.

The fact that Figs. 8, 11, and 12 are so similar suggests that the footprint structure is determined by the passage of the trajectories through the stochastic layer but does not depend on the detailed structure of the plasma edge. This passage leads to a scattering process which can be considered as having three phases, which are depicted schematically in Fig. 13. We focus on a single trajectory that initially lies well within the stochastic layer and follow its evolution in the poloidal $x-z$ plane. In the first phase of evolution the trajectory is confined within the stochastic layer, making many toroidal (and hence also poloidal) circuits. The first phase is declared to end when the trajectory crosses $x=0$ for the last time before intersecting a plate. 
The second phase of evolution takes the trajectory from this last crossing of $x=0$, across the stable/unstable manifold corresponding to the perturbed separatrix (plasma edge), and ends at the point of closest approach of the trajectory to the perturbed $\mathrm{x}$-point near the active divertor plate. The distance between the the $\mathrm{x}$-point and the point of closest approach represents the impact parameter of the scattering process. The third phase of evolution takes the trajectory from the $\mathrm{x}$-point region to the divertor plate.

The above picture of events implies that the stochastic spreading which makes the whorl "fuzzy" is due to the chaotic scattering of trajectories. Since the distribution of $N_{\phi}$ values with respect to $\phi_{0}$ is effectively random, the poloidal location of the crossing point of the trajectory with the plasma edge is also random. This leads to a random scattering impact parameter.

The picture also allows a calculation of the relative size of the magnetic foorprints on the upper and lower divertor plates. For this we integrate the field line equation $9 \mathrm{~b}$ over the Phase 2 mution after expanding about the unperturbed separatrix. Defining $\Delta_{+}$and $\Delta_{-}$to be the maximum possible impact parameters for the lower and upper perturbed x-points, respectively, then,

$$
\Delta_{ \pm} \approx R_{0} \frac{\epsilon}{B_{0}} \exp \left( \pm \dot{a} / R_{0}\right) \int_{ \pm} \cos \phi d \phi,
$$

where the $\phi$ integrations are over the limiting orbits which give rise to the maximum impact parameters. Since these maxima are defined with respect to all possible orbits, we can take the same $\phi$ values for the integrations. Then

$$
\frac{\Delta_{+}}{\Delta_{-}} \approx \exp 2 a / R_{0} .
$$

To relate the maximum impact parameters to the maximum footprint widths, we use the flux expansion relationship

$$
\frac{\Delta x_{p}^{+}}{\Delta x_{p}^{-}} \approx\left(\frac{\Delta_{+}}{\Delta_{-}}\right)^{2} .
$$

Thus,

$$
\frac{\Delta x_{p}^{+}}{\Delta x_{p}^{-}} \approx \exp 4 a / R_{0} \approx 3.3
$$

is an estimate for the ratio of the width of the footprint on the lower plate to the width on the upper plate. This agrees well with the numerical results shown in the figures. 
This calculation suggests that the width of the footprint scales linearly with the perturbation amplitude. This is verified by our numerical calculations.

\section{Discussion}

In the presence of $n=1$ nonaxisymmetric magnetic field perturbations, the footprint of the field line tractories on a divertor plate assumes a spiral structure, shown in Fig. 11. We have shown that the existence of this spiral structure follows under quite general assumptions, and does not depend on the particular three-wire model that we have investigated numerically. For field errors of the magnitude that exist in present day tokamak devices, and are contemplated for the next generation of tokamaks, the radial width of this structure is comparable to the width of the scrape off layer. The nonaxisymmetric perturbations will be larger in the presence of locked modes, producing spiral structures of correspondingly larger dimension. (The radial width of the structure scales linearly with the perturbation amplitude.)

One striking feature of this calculation is that the footprint exhibits multiple peaks as a function of $R$ at a fixed value of $\phi$. Experimental measurements of the heat deposition profile on the DIII-D divertor plates do exhibit a double peak of this sort in the presence of locked modes.

Cross-field diffusion can be expected to wash out the details of the spiral footprint structure, so that it only approximately represents the heat deposition profile. The extent to which the small scale structure is washed out will be determined by the ratio of the scrape-off width to the width of the spiral structure. This is consistent with the observation of bifurcated heat deposition profiles in DIII-D only in the presence of locked modes. Larger tokamaks, with smaller scrape-off widths, can be expected to preserve more of the structure, producing more localized hot spots.

The nonaxisymmetric structure of the footprint on the divertor plate can be expected to lead to nonaxisymmetric heat deposition. Even though much of the structure is washed out by cross-field diffusion in the absence of locked modes on present day tokamaks, we might expect some residual nonaxisymmetry. The toroidal dependence of the heat deposition has been measured on Asdex, ${ }^{16}$ and nonaxisymmetric deposition has been observed even in the absence of locked modes. 
In larger tokamaks, with smaller scrape-off widths, we can expect a greater nonaxisymmetry in the heat deposition, giving a larger heat load peaking factor. This poses a potential problem for the next generation of tokamaks, where heat load on the divertor plates is already an issue.

Finally, we remark that stochasticity in the neighborhood of the divertor separatrix is sometimes proposed as a method for spreading the heat load on the divertor plates. We have found that for $n=1$ magnetic field perturbations there is a significant region of chaotic field line trajectories outside the last closed flux surface. Little of this chaotic structure is reflected in the divertor footprint. For pure $n=1$ perturbations, the stochastic broadening is small compared to the scale of the coherent nonaxisymmetric structure.

\section{ACKNOWLEDGMENT}

We are grateful for useful discussions with A. Boozer, B. Braams, T. Evans, R. Goldston, R. LaHaye, H. Neilson, and D. Stotler. This work was supported by the U.S. Department of Energy Contract \# DE-AC02$76 \mathrm{CHO} 3073$. 


\section{Appendix. Model Illustrating Formation of the Spi- ral Pattern}

In this Appendix we prest it a simple model that illustrates the development of a closed curve into the spiral pattern known as a whorl. ${ }^{15}$ For simplicity we consider a system whose flux surfaces are circles, whose rotational transform vanishes at the chosen flux value $\psi=1.0$ (representing a separatrix), and whose shear is constant. The system dynamics which governs the evolution of a point with initial coordinates $\left(\psi_{k}, \phi_{k}\right)$ is defined to be

$$
\begin{gathered}
\psi_{k+1}=\psi_{k}, \\
\phi_{k+1}=\phi_{k}+k
\end{gathered}
$$

where

$$
{ }_{k}=2 \pi\left(\psi_{k}-1.0\right) / 5.0
$$

Figure 14 shows the result of iterating the model equations for initial conditions which lie on a shifted circle of radius 1.5 , tangential to the separatrix at $\phi=\pi$. The separatrix is indicated by a dashed circle, the initial conditions are by open circles. Successive $k$ iterates of the equations lead to the points represented by the symbols $*, x$, and + , respectively. These are seen to lie on a developing spiral whose innermost point corresponds to the minimum transform. For the model in this Appendix this minimum transform is $=0$. The similarity of the spiral pattern shown in Fig. 14 with the pattern developed in Fig. 9 and Fig. 10 is clearly evident. 


\section{References}

${ }^{1}$ R. J. Goldston, G. H. Neilson, D. B. Batchelor, G. Bateman, M. G. Bell, D. N. Hill, W. A. Houlberg, S. C. Jardin, S. S. Medley, N. Pomphrey, M. Porkolab, R. O. Sayer, D. J. Sigmar, D. P. Stotler, D. J. Strickler, M. Ulrickson, R. E. Waltz, P. T. Bonoli, B. Braams, J. Brooks, M. D. Carter, H. F. Dylla, R. C. Englade, R. H. Goulding, J. R. Haines, D. J. Hoffman, J. C. Hosea, E. F. Jaeger, J. L. Johnson, S. M. Kaye, C. Kessel, J. Kinsey, A. Kritz, R. J. LaHaye, R. A. Langley, J. Manickam, T. K. Mau, J. Milovitch, W. A. Peebles, C. K. Phillips, R. Pillsbury, R. Prater, A. Reiman, T. Rognlien, P. M. Ryan, J. E. Scharer, C. E. Singer, G. R. Smith, R. D. Stambaugh, D. W. Swain, J. S. Tolliver, J. R. Wilson, K. Wilson, K. M. Young, and J. J. Yugo, Burning Plasma Experiment Design Description, Fusion Technology (to be published in Fusion Technology, 1992).

${ }^{2}$ D. E. Post, K. Borrass, J. D. Callen, S. A. Cohen, J. G. Cordey, F. Engelmann, N. Fujisawa, M. F.Harrison, J. T. Hogan, H. J. Hopman, Y. Igitkhanov, O. Kardaun, S. M. Kaye, S. Krasheninnikov, A. Kukushkin, V. Mukhovatov, W. M. Nevins, A. Nocentini, G. W. Pacher, H. D. Pacher, V. V. Parail, L. D. Pearlstein, L. J. Perkins, S. Putvinskij, K. Riedel, D. J. Sigmar, M. Sugihara, D. W. Swain, T. Takizulia, K. Tani, T. Tsunematsu, N. A. Uckan, J. G. Wegrowe, J. Wesley, S. Yamamoto, R. Yoshino, K. Young, P. N. Yushmanov, and International Contributors, ITER Physics, ITER Documentation Series No. 21, IAEA Vienna, 1991

${ }^{3}$ P. C. Stangeby and G. M. McCracken, Nucl. Fusion 30, 1225 (1990).

${ }^{4}$ A. J. Lichtenberg and M. A. Lieberman, Regular and Stochastic Motion, Springer-Verlag, New York Heidelberg Berlin, (1983).

${ }^{5}$ B. Eckhardt, Physica D 33, 89 (1988).

${ }^{6}$ Y-T. Lau and J. M. Finn, Astrophys. J. 366, 577 (1991)

${ }^{7}$ Y. Tomita, S. Seki, and H. Momota, J. Phys. Soc. Jpn. 42, 687 (1977).

${ }^{8}$ A. H. Boozer and A. B. Rechester, Phys. Fluids 21(4), 682 (1978).

${ }^{9}$ R. J. LaHaye, Nucl. Fus. 31, 1550 (1991). 
${ }^{10}$ D. N. Hill, T. Petrie, M. Mahdavi, L. Lao, W. Howl, Nucl. Fusion 28(5), 902 (1988).

${ }^{11}$ J. A. Snipes, D. J. Campbell, T. C. Hender, M. V. Hellermann, and H. Weisen, Nucl. Fusion 30(2), 205 (1990).

${ }^{12}$ H. Zohm, A. Kallenbach, H. Bruhns, G. Fussmann, and O. Klueber, Europhys. Lett. 11(8), 745 (1990).

${ }^{13}$ R. J. L. Haye and J. T. Scoville, Rev. Sci. Inst. 62, 2146 (1991).

${ }^{14}$ M. Abramowitz and I. Stegun, editors, Handbook of Mathematical Functions, chapter 9, page 376, National Bureau of Standards, Washington, D. C., 1964.

${ }^{15}$ M. V. Berry and N. L. Balazs, J. Phys. A: Math. Gen. 12(5), 625 (1979).

${ }^{16}$ T. E. Evans, J. Neuhauser, F. Leuterer, E. R. Muller, and The ASDEX Team, J. Nucl. Mater. 176, 202 (1990). 


\section{Figures}

FIG. 1. Structure of tokamak magnetic surfaces in the presence of an axisymmetric poloidal divertor. $I_{p}$ and $I_{a}$ indicate the straight line current flaments used in our three-wire model.

FIG. 2. Separatrix elongation, $\kappa_{N}$, as a function of wire current ratio, $\gamma=$ $I_{c} / I_{p}$, for 3-wire model.

FIG. 3. Safety factor, $q$, as a function of minor radius on midplane near the separatrix.

FIG. 4. Surface of section for field line integrations without divertor plates for $\epsilon / B_{0}=1 \times 10^{-3}$. (a) $\phi=0$, and (b) $\phi=8 \pi / 5$.

FIG. 5. Expanded view of lower divertor region of Fig. 4.

FIG. 6. Surface of section $x$ vs. $\phi$ at $z_{p}=-1.40$ for $\epsilon / B_{0}=1 \times 10^{-3}$.

FIG. 7. Number of toroidal transits, $N_{\phi}$, before reaching a plate, as a function of initial $x$ and $\phi$.

FIG. 8. Magnetic footprint of 2000 field line intersections with the (a) lower and (b) upper divertor plates for initial conditions inside the plasma region using Eq. (13).

FIG. 9. Magnetic footprint on the lower divertor plate for 2000 field lines lying on initial curves defined by Eq. (14) with (a) $\delta x_{0} / a=0.014$ and (b) $\delta x_{0} / a=0.0125$.

FIG. 10. Magnetic footprints on the (a) lower and (b) upper divertor plates for $\delta x_{0} / a=0.012$. (c) $N_{\phi}$ vs. $\phi_{0}$ for this set of initial conditions.

FIG. 11. Magnetic footprints on the (a) lower and (b) upper divertor plates for $\delta x_{0} / a=0.007$. (c) $N_{\phi}$ vs. $\phi_{0}$ for this set of initial conditions.

FIG. 12. Magnetic footprints on the (a) lower and (b) upper divertor plates for $\delta x_{0} / a=0.002$. (c) $N_{\phi}$ vs. $\phi_{0}$ for this set of initial conditions.

FIG. 13. Cartoon of the three phases of evolution of a trajectory between the stochastic layer and the divertor plate. 
FIG. 14. Development of spiral whorl for initial conditions on a circle, shifted with respect to flux surfaces. 


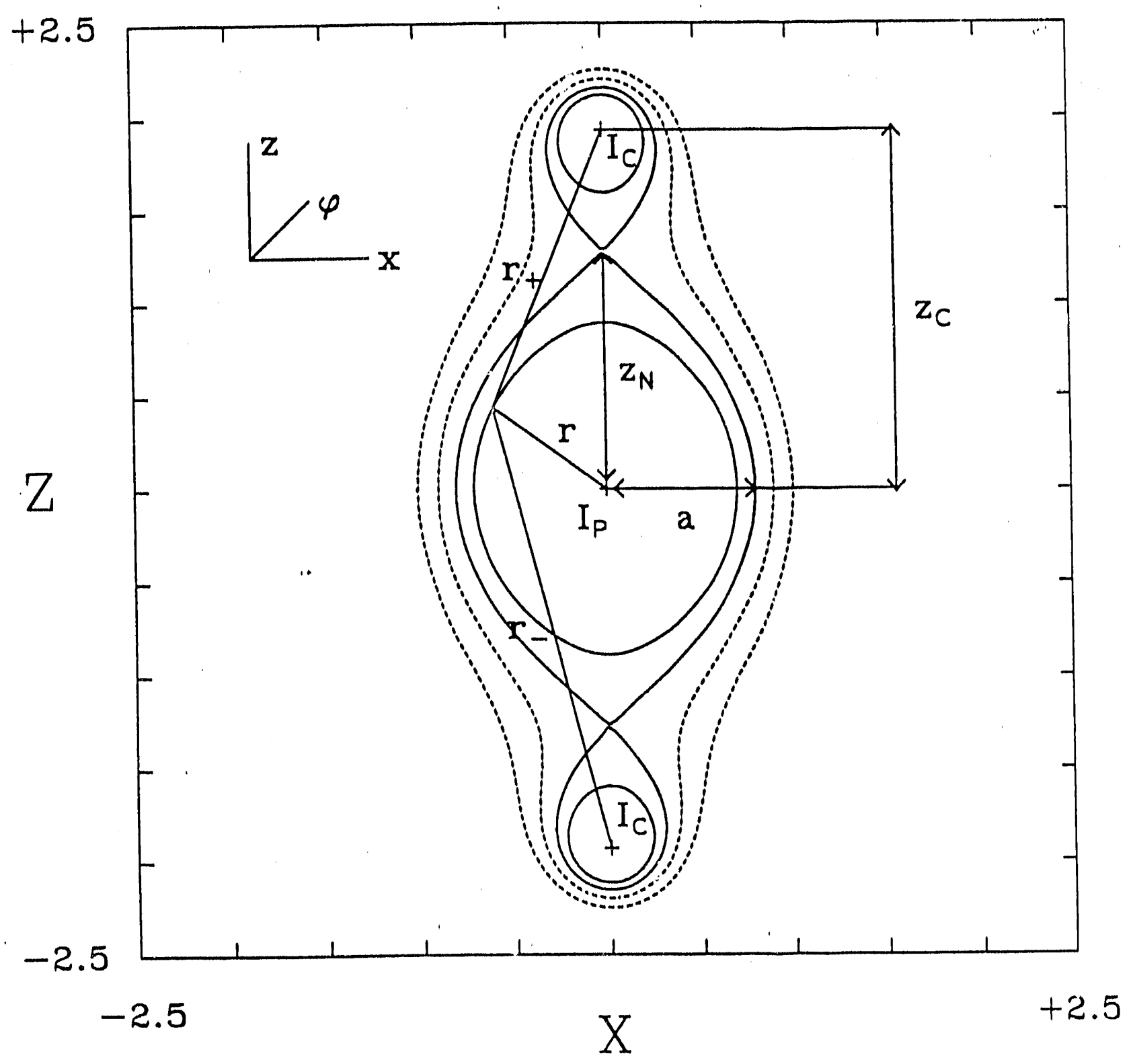

Fig. 1 


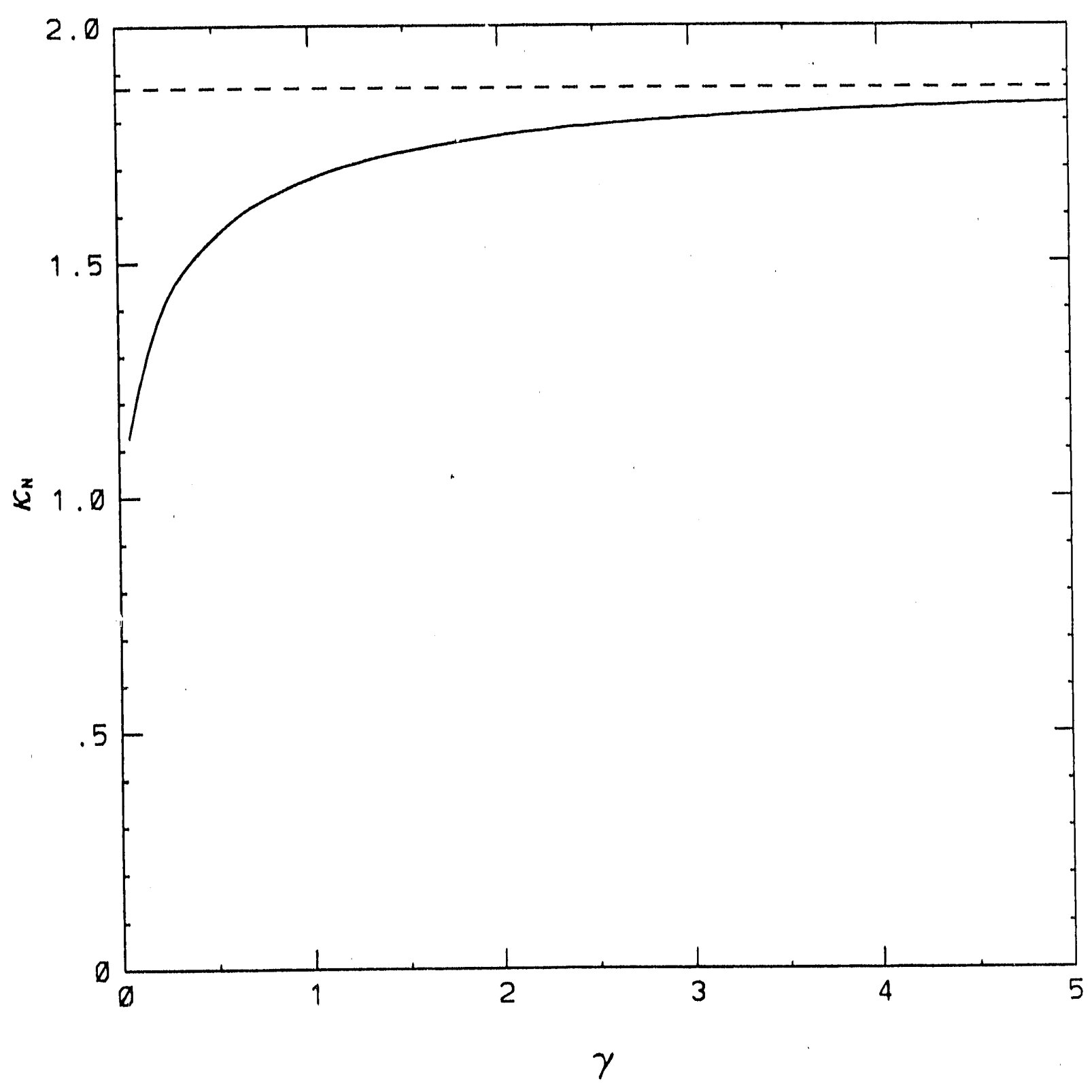

Fig. 2 


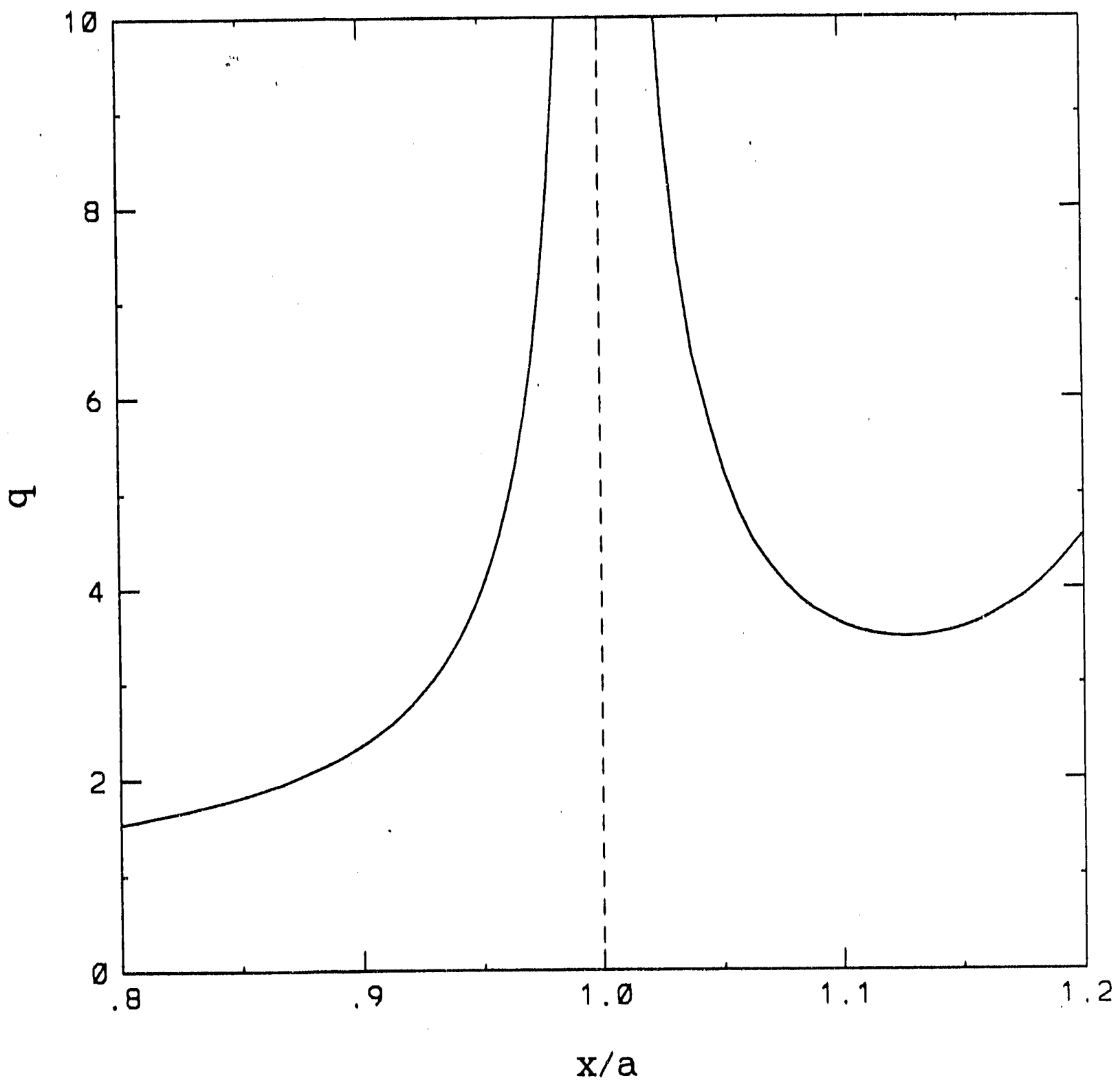

Fig. 3 
$91 T 1031$

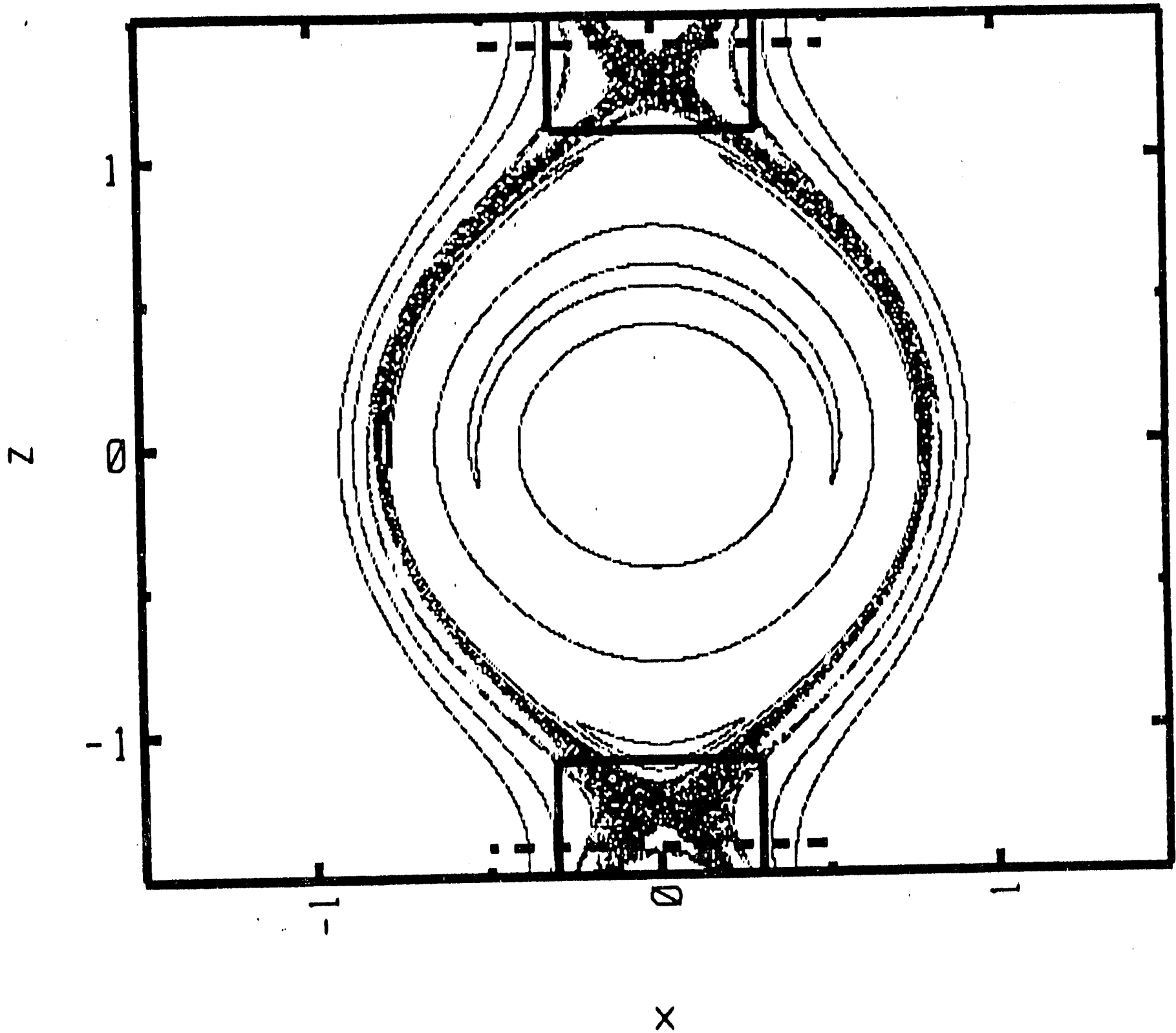

Fig. $4 a$ 
91 T 1026

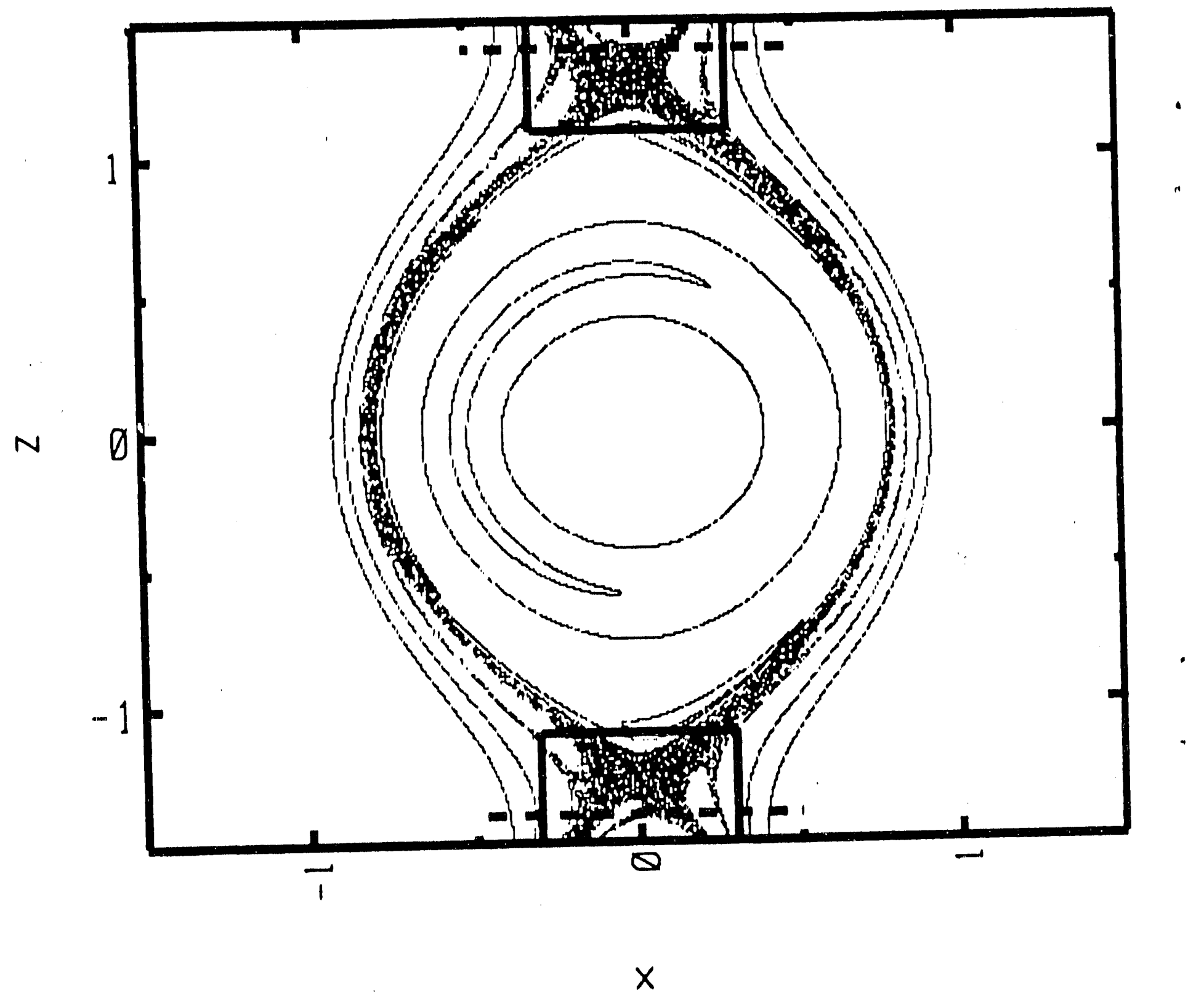

Fig. $4 b$ 


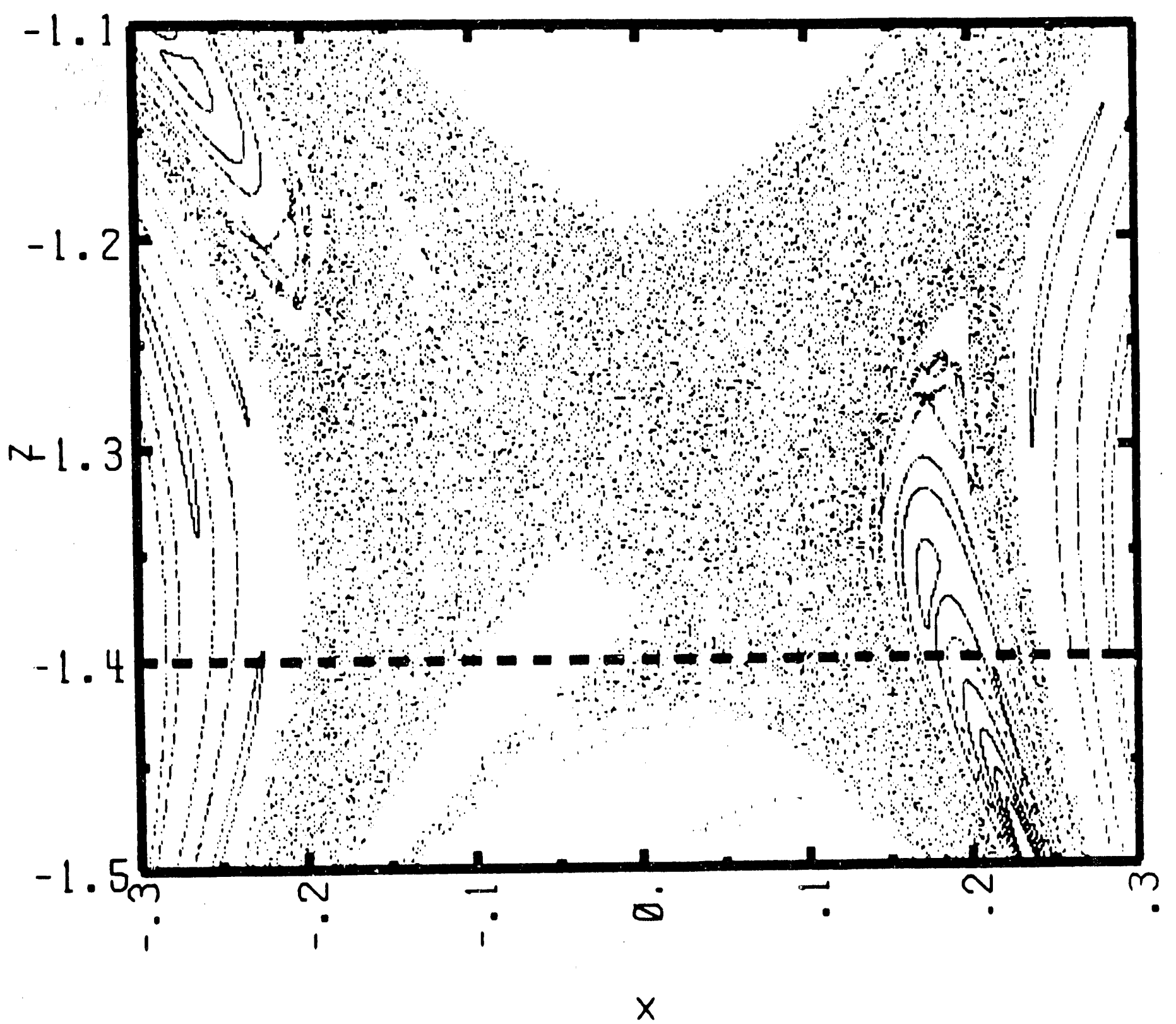

Fig. 5 


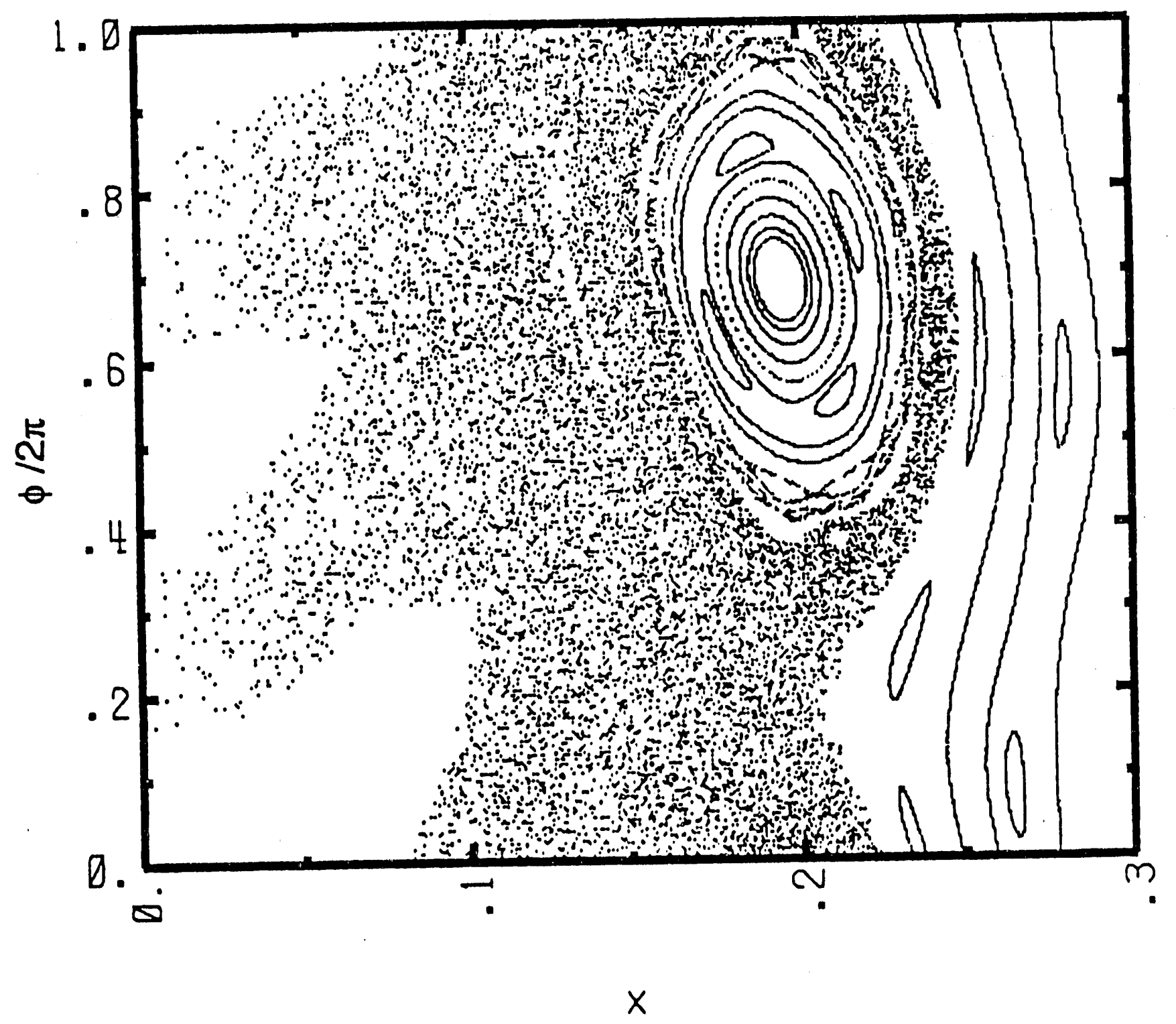

Fig. 6 


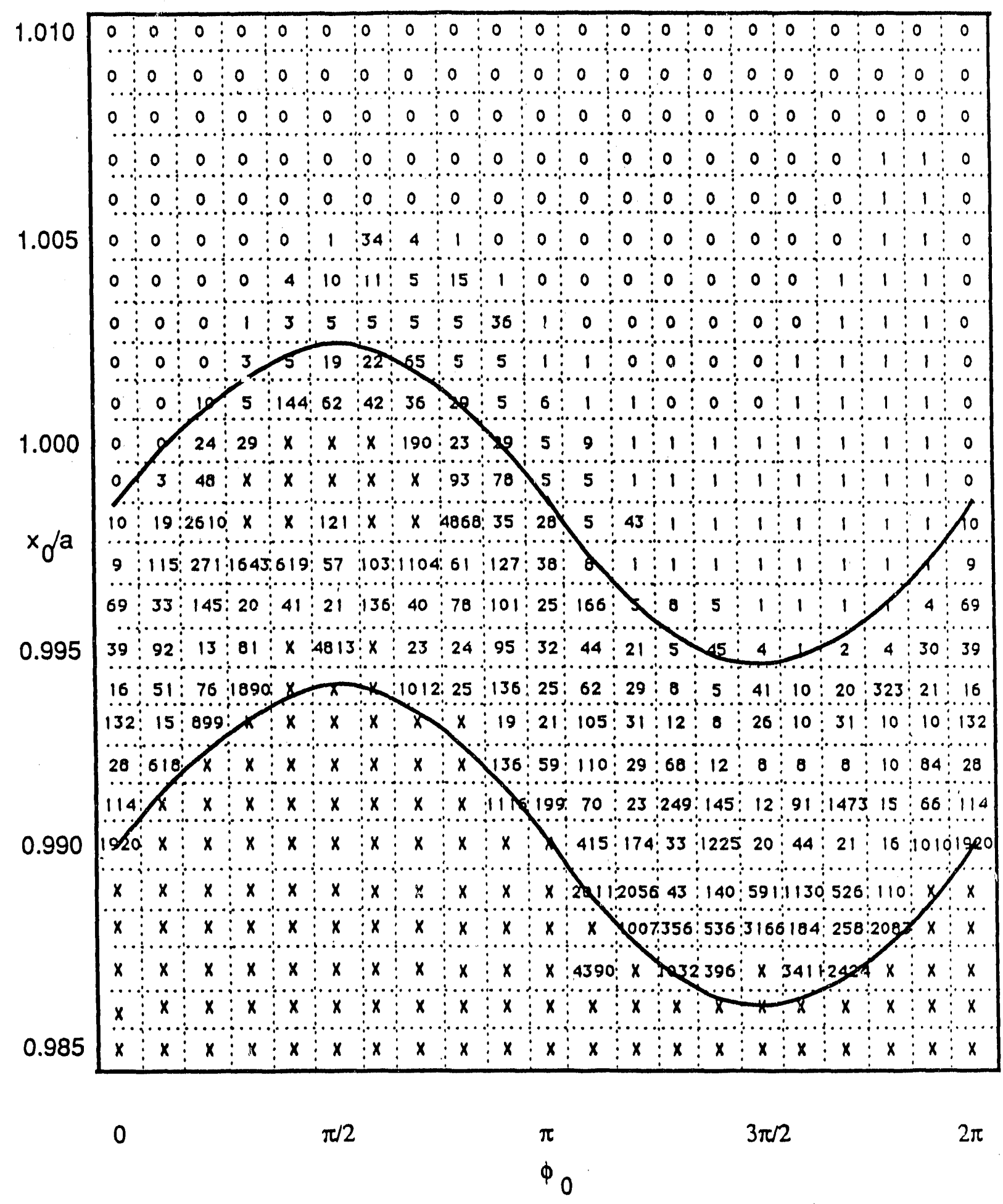

Fig. 7 


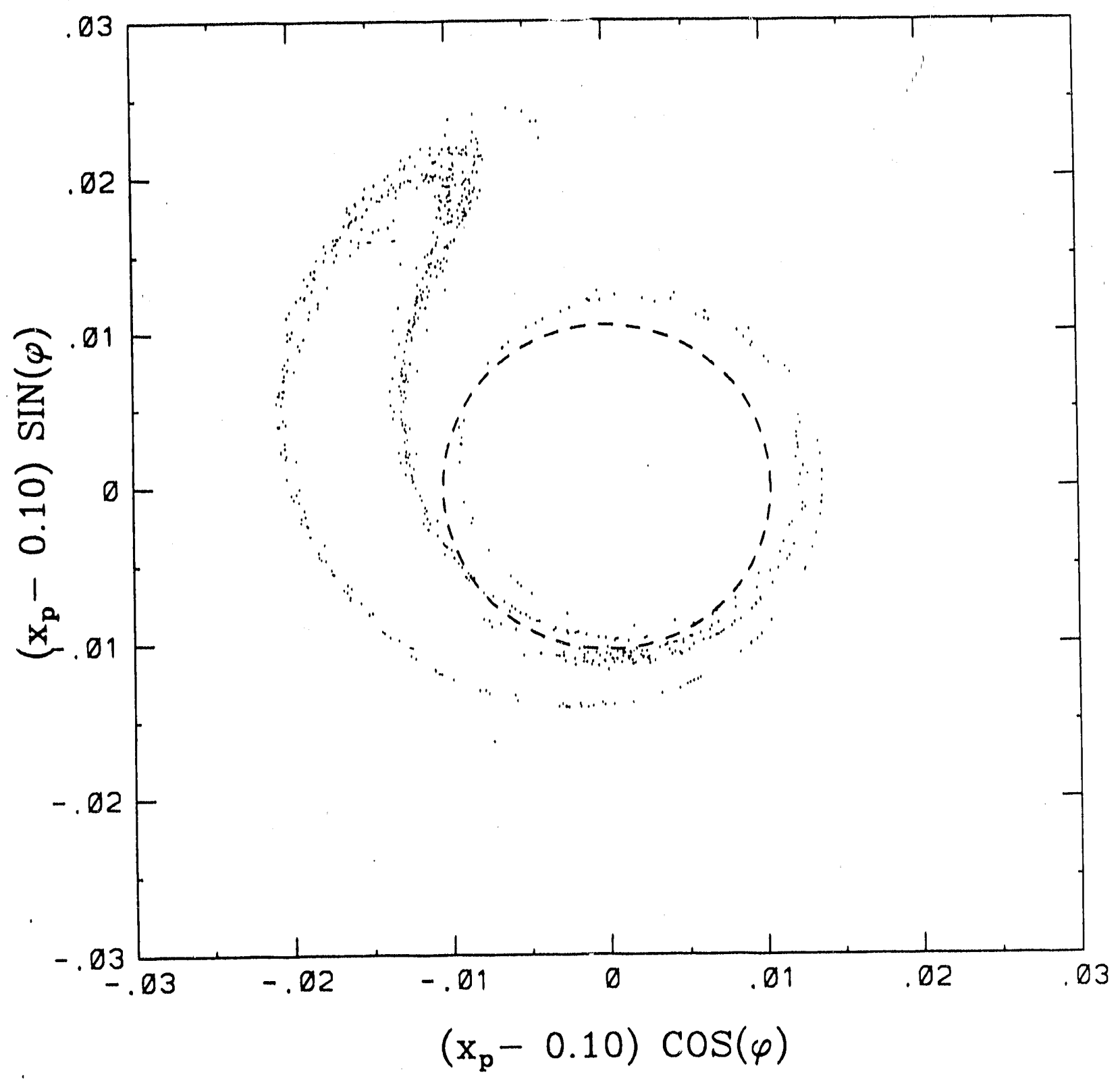

Fig. 8a 


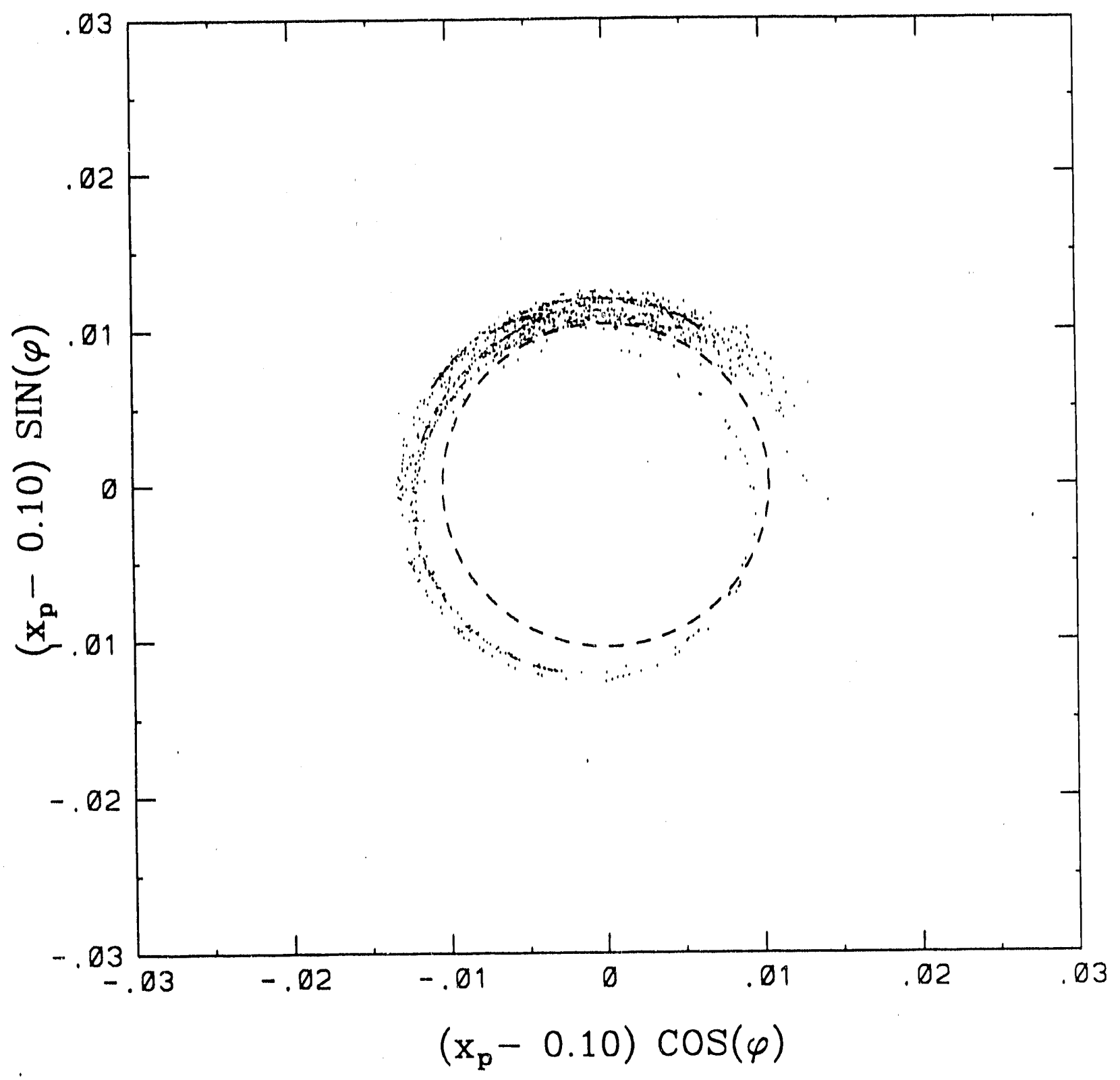

Fig. $8 b$ 


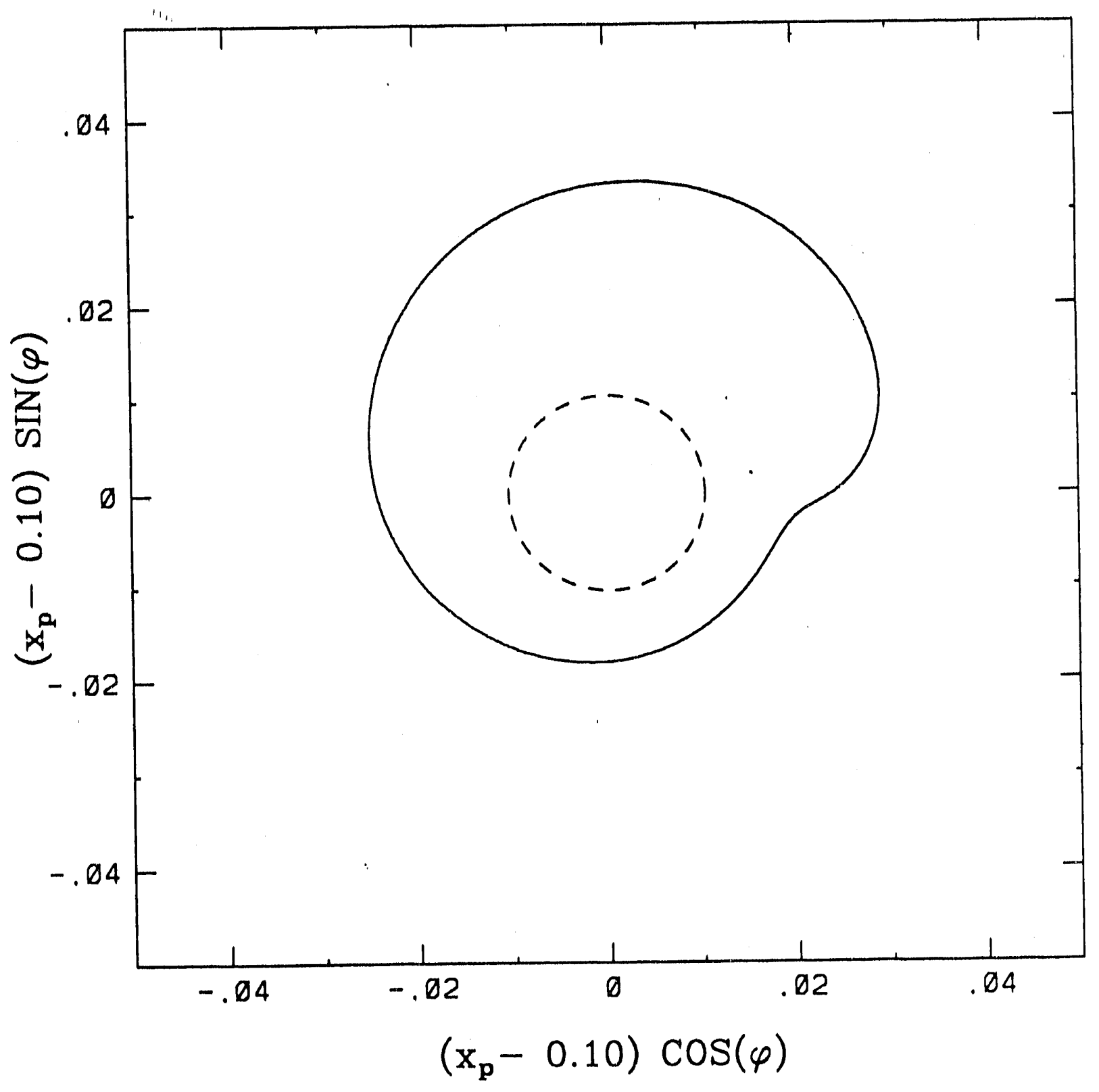

Fig. 9a 


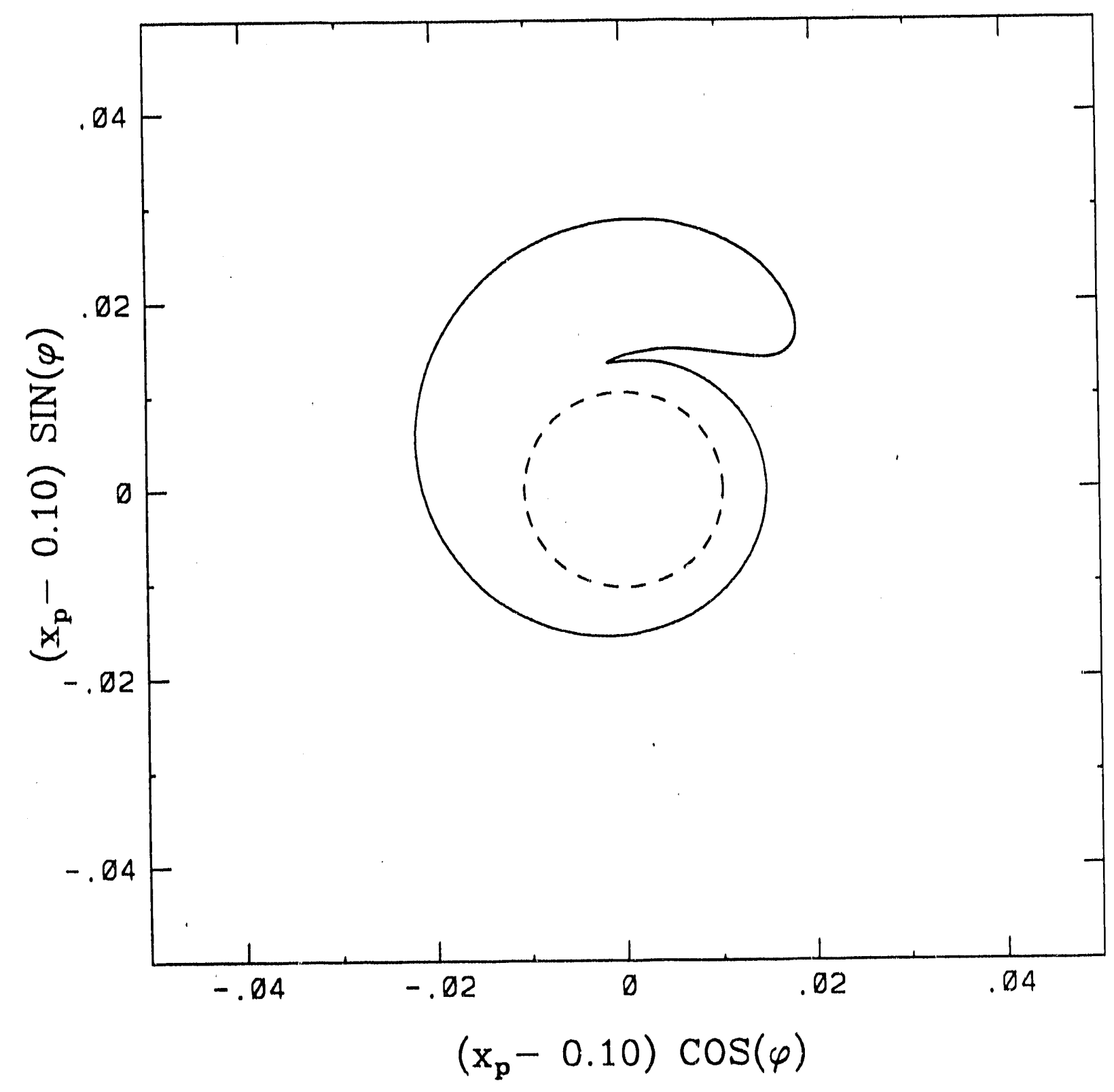

Fig. 96 


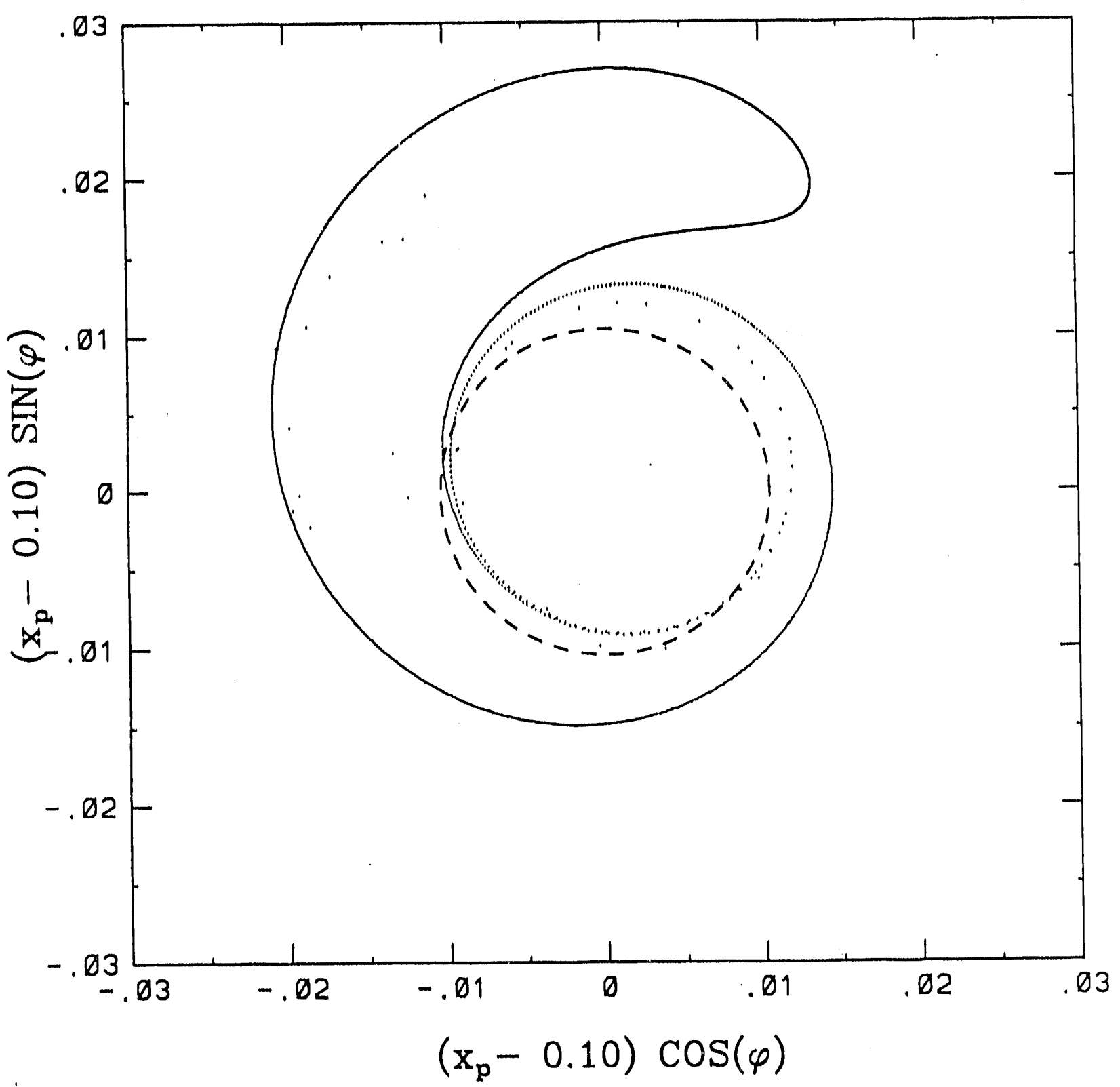

Fig. 10a 


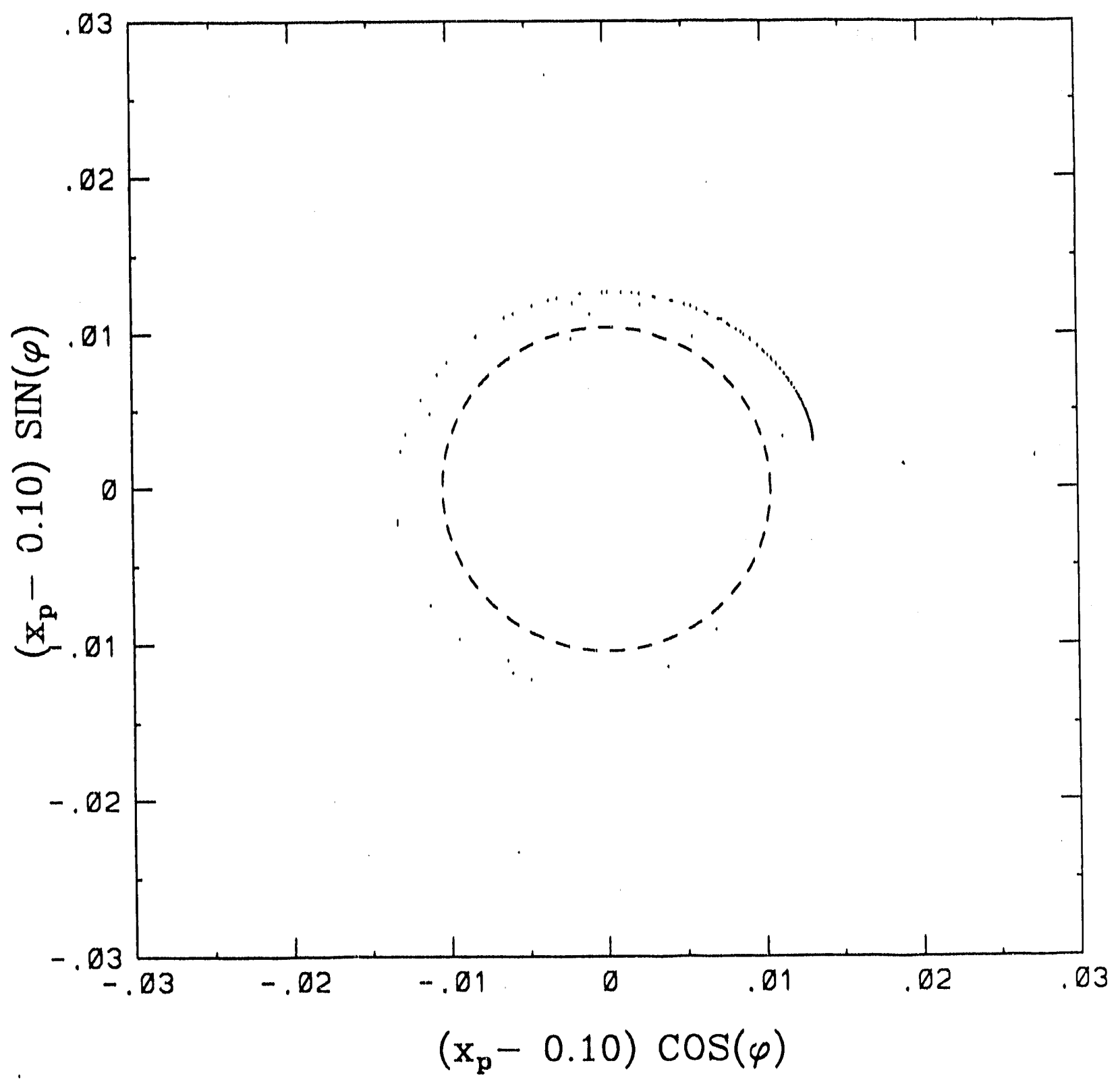

Fig. $10 b$ 


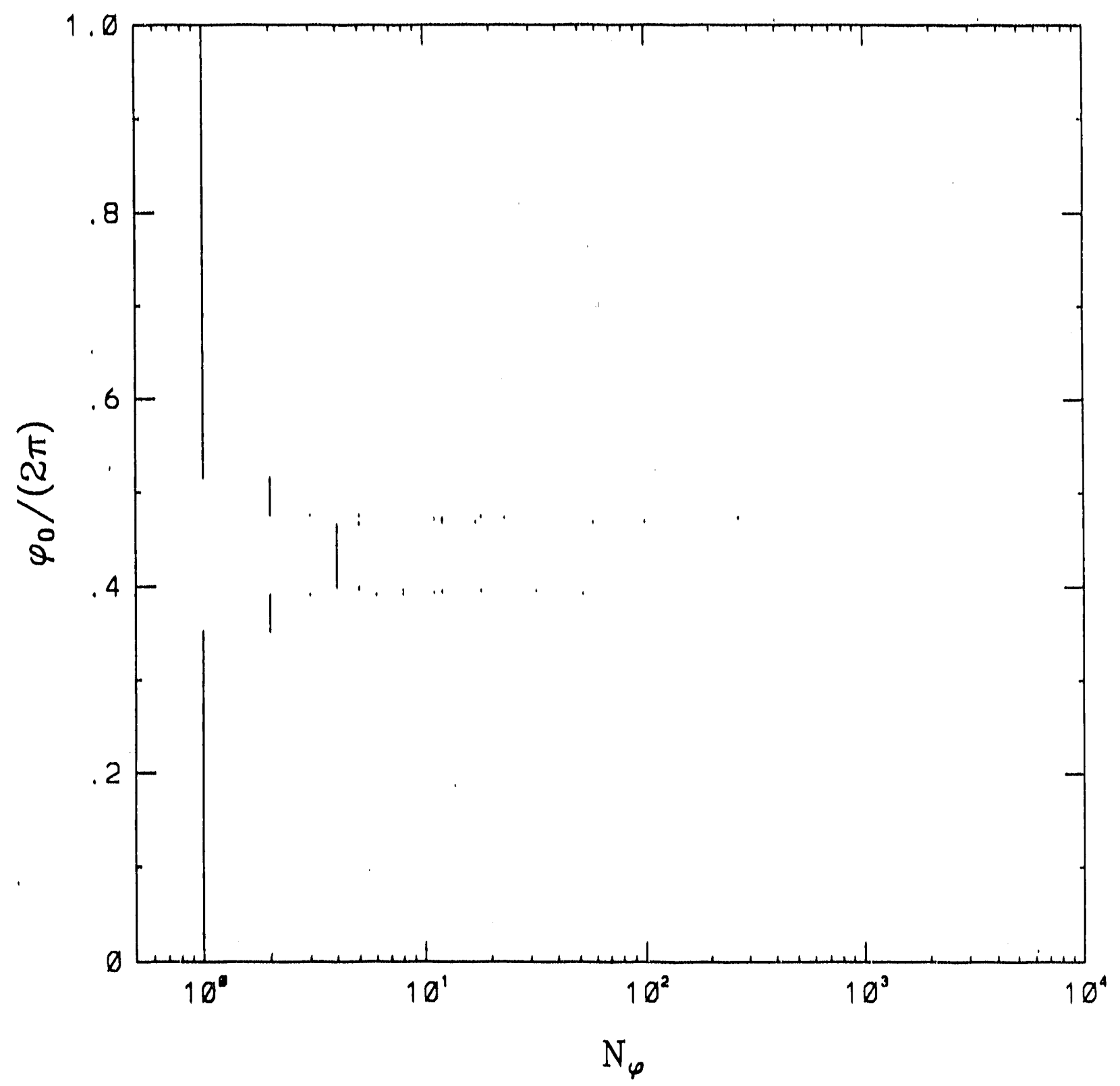

Fig. $10 c$ 


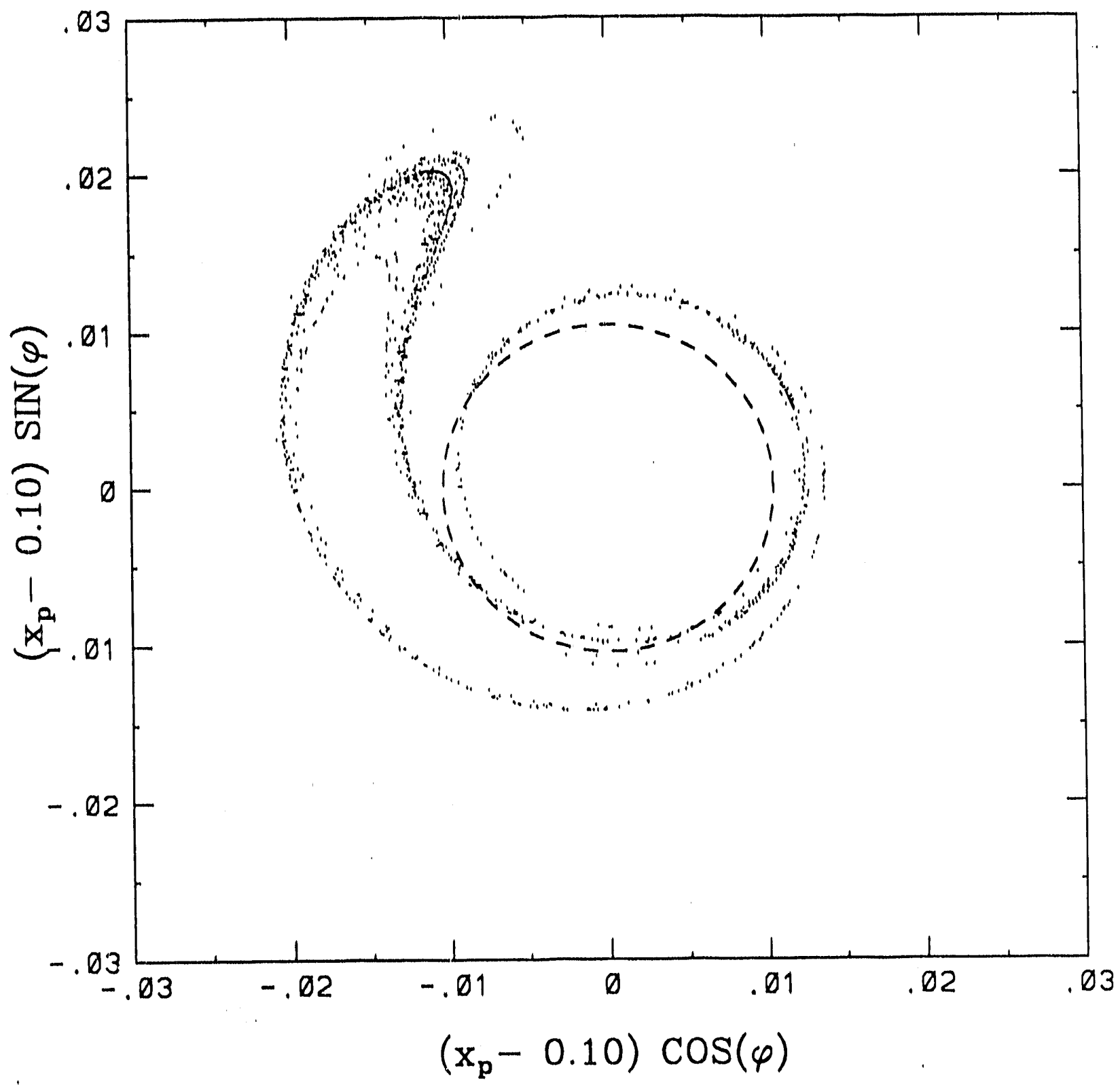

Fig. 11a 


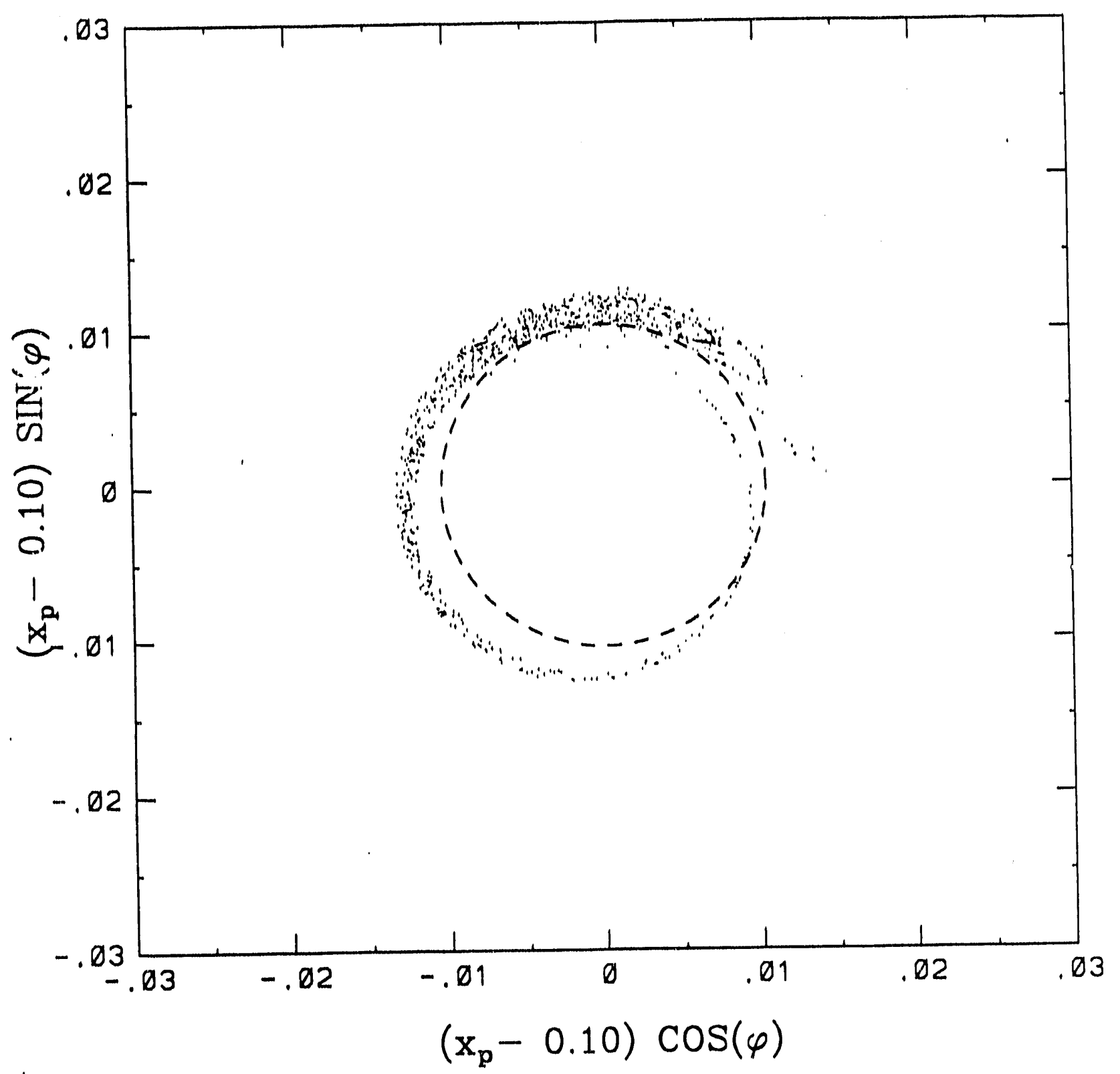

Fig. 11b 


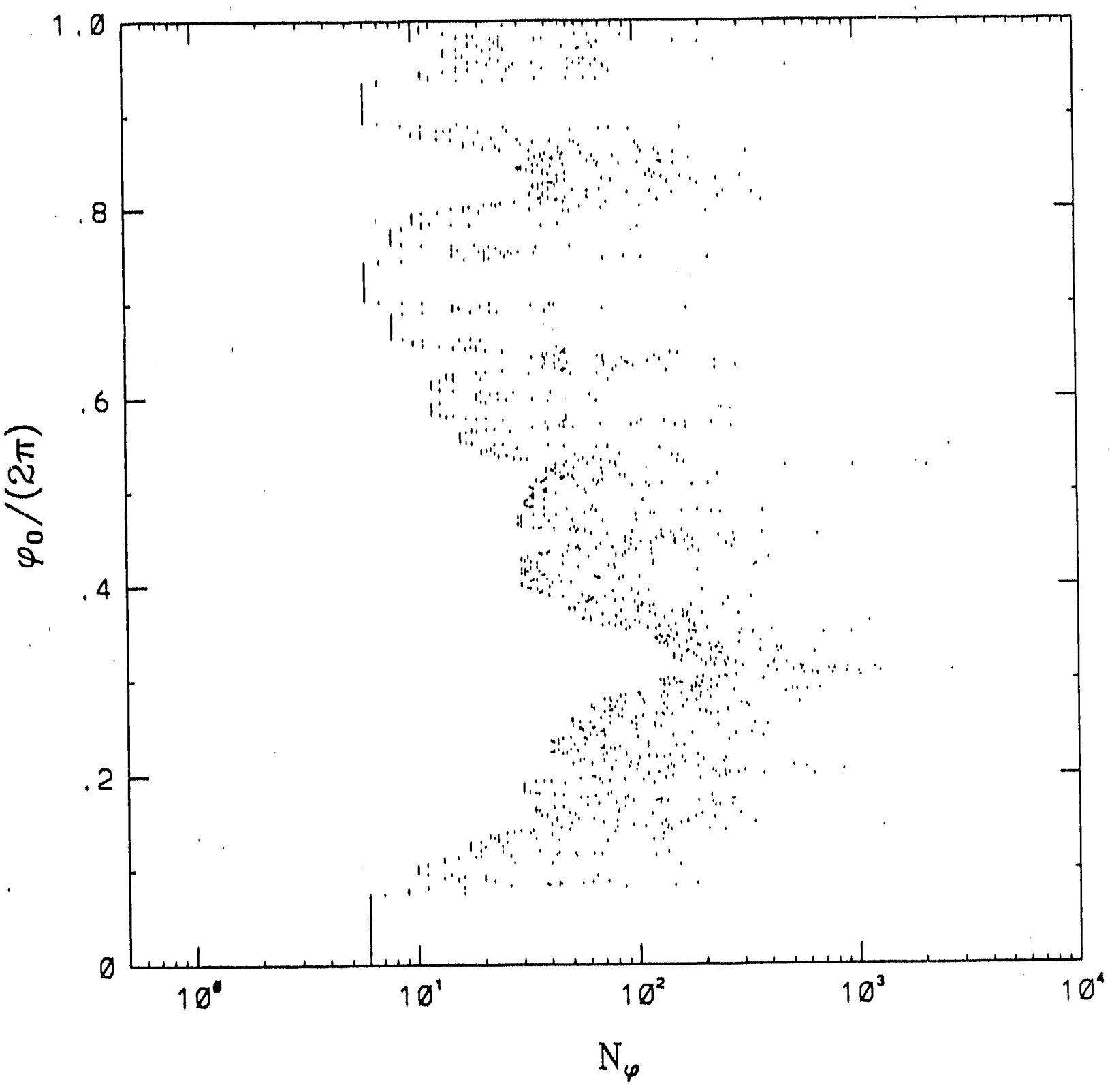

Fig. $11 \mathrm{c}$ 


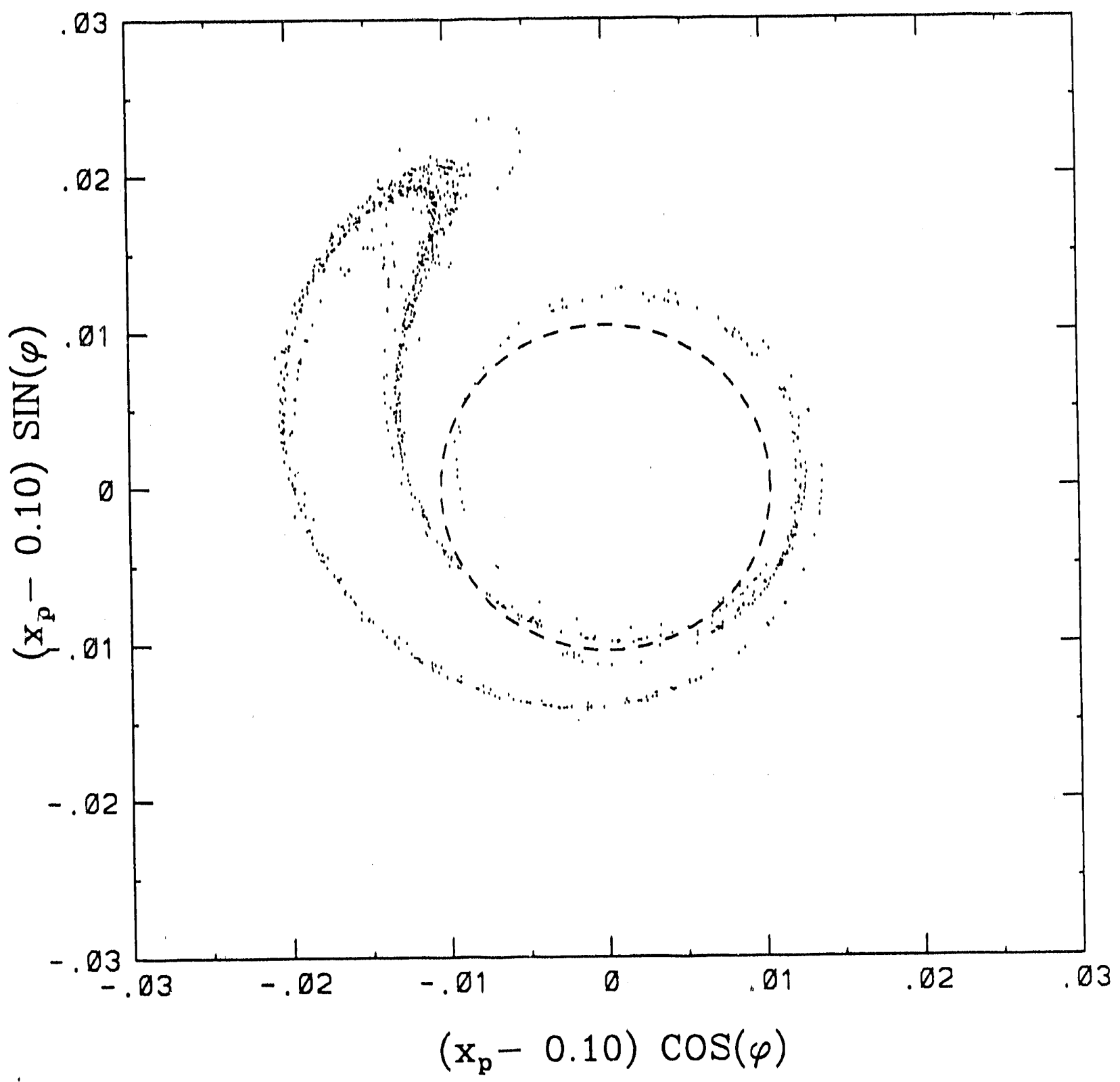

Fig. $12 a$ 


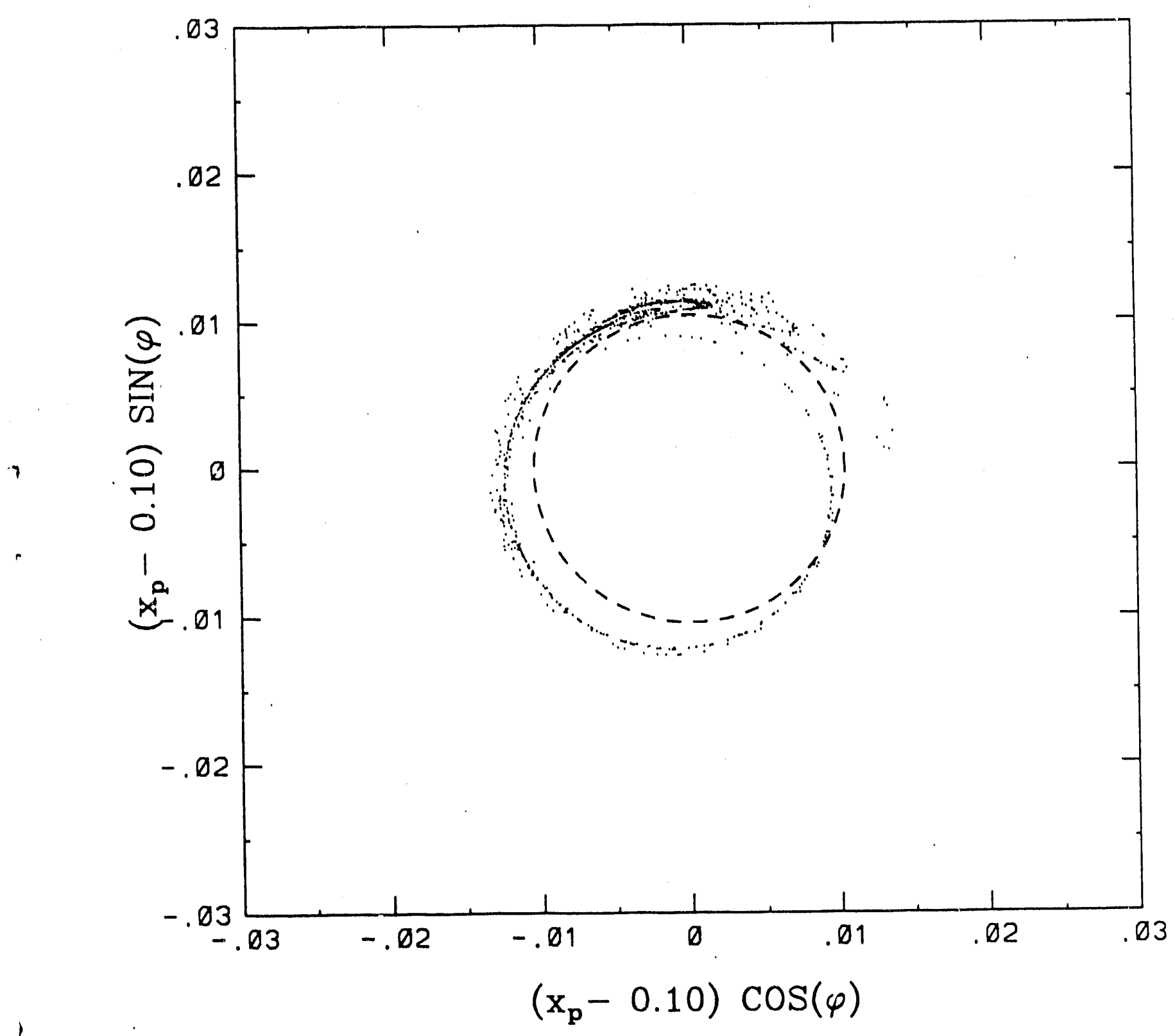

Fig. $12 b$ 


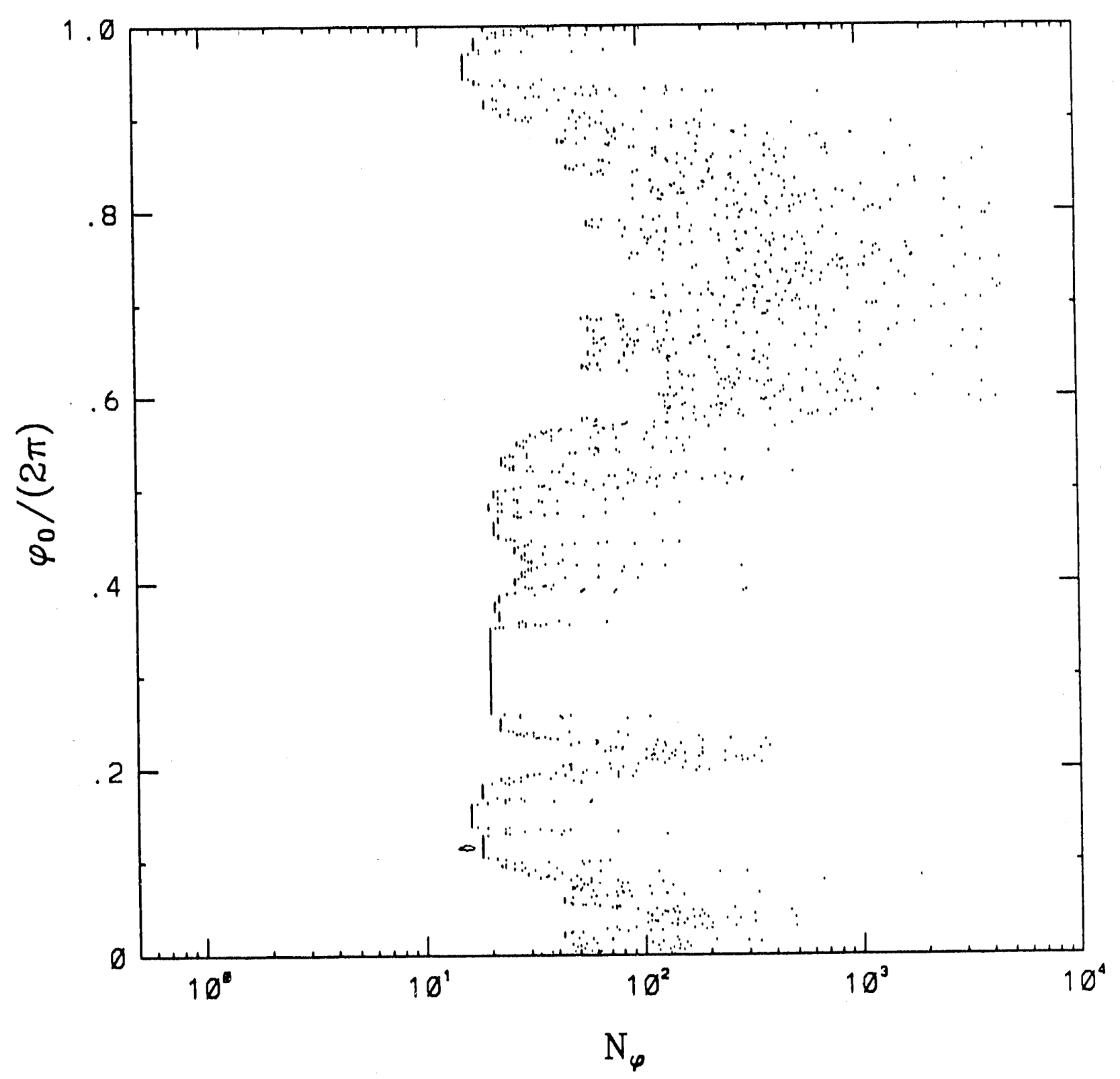

Fig. $12 c$ 


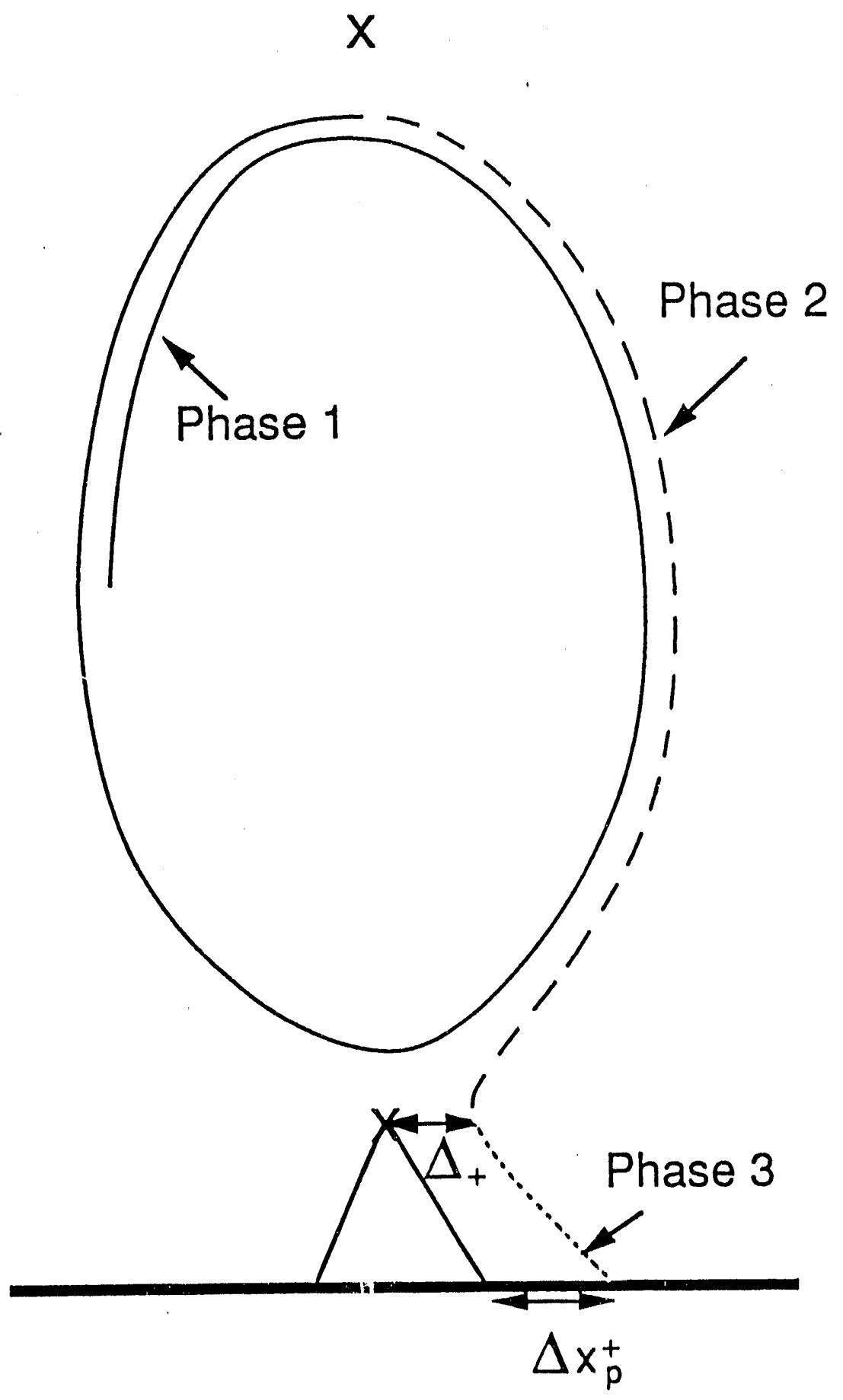

Fig. 13 


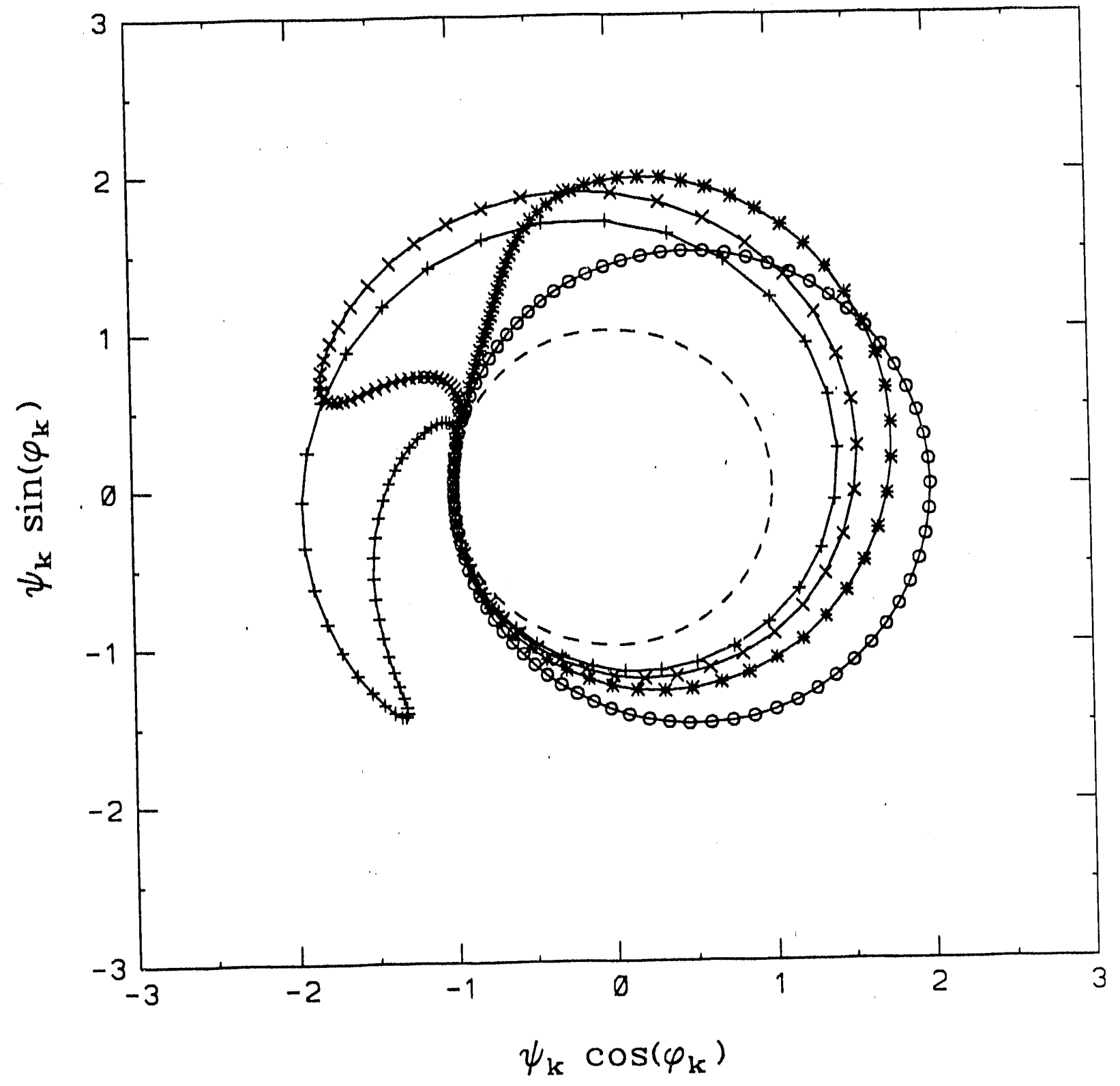

Fig. 14 
Dr, F. Paolon, Univ, of Wollongong, AUSTRALIA

Prof. M.H. Brennan, Univ, of Sydney, AUSTPALIA

Plasma Rosearch Lab, Austrilen Nat. Univ., AUSTRALIA

Prof. I.R, Jones, Finders Univ, AUSTRALIA

Prof, F. Cap, Inst for Theoretical Physics, AUSTRIA

Prot. M. Hoindor, Insditut far Theoretisctio Physik, AUSTRIA

Prot. M. Gooseons, Astronomisch Instbust, BELGIUM

Ecole Royelo Nilltuire, Leb. do Phy. Plaemas, BELGIUM

Commisuion-Europeen, DG. XII-FUsion Prog., BELGIUM

Prof. R. Boudqut, Rikeuniveritin Gent, BELGIUM

Dr. P.H. Sakenaka, Instuwo Fiaka, BRAZIL.

Insttuto Nedond Do Ponquieas Espeodals-INPE, BRUZIL

Documenta Orifos, Atomic Eneroy of Ceneda Lid., CANADA

Dr. M.P. Bectyrnekd, MPB Tectiriologios, Inc., CANADA

Dr. H.M. Skaregard, Univ, of Seckatchewen, CANADA

Prot. J. Tolctumann, Univ, of Montreal, CANADA

Prot. S.R. Sreentvecen, Univ, of Celoary, CANADA

Prof. T.W. Johnston, INAS-Energio, CANADA

Dr. R. Botton, Contre canacien de hision megnatque, CANADA

Dr, C.R. James., Univ. of Aberta, CANADA

Dr. P. Lukte, Kornenskeho Universzita, CZECHO-SLOVAKIA

The Librarian, Cutham Laborabry, ENGLAND

Library, R61, Authertord Applotion Laboratory, ENGLAND

Mre. S.A. Hutchineon, JET Librery, ENGLAND

Dr. S.C. Shemen, Univ, of South Pectic, FIJI ISLANDS

P. Mathonen, Univ. of Heisiniki, FINLAND

Prof. M.N. Buseac, Ecolo Polytochnique, FRANCE

C. Moutiot, Lab. de Physiquo dos Milioux loniads, FRANCE

J. Radah, CENCADARACHE - Bat 506, FRANCE

Prot. E. Economou, Univ, of Croto, GREECE

Ms. C. Pinni, Univ, of loamina, GREECE

Dr. T. Nuel, Acadermy Blbliographic Ser., HONG KONG

Preprint Lbbrary, Hungarian Acadomy of Sal. HUNGARY

Dr. B. DasGupta, Saha inst, of Nucteer Physica, INDIA

Dr. P. Kaw, Inst. for Plasma Poscarch, INDIA

Dr. P. Roweneu, leraed inst, of Tectnology, ISPUEL

Libraien, Intomations Contor for Theo Phyeics, ITALY

Miss C. Do Palo, Aseociazione EURATOMENEA, ITALY

Dr. G. Groseo, Istruto di Fieica del Plasma, ITALY

Prot. G. Rostengoni, Isthuto Gas lonizzati Det Cnr, ITALY

Dr. H. Yamato, Tochiba Res a Dowel Cenbr, JAPAN
Prot. I. Kawakemi, Hiroshima Untv, JAPAN

Prol, K. Nishlkawa, Hhoshima Univ., JAPAN

Director, Jepen Alomic Enorgy Research Inst, JAPAN

Prof. S. Itoh, Kyushu Univ., JAPAN

Reseanch Into, Ctr., National instit. for Fusion Sclence, JAPAN

Prot. S. Tenaka, Kyoto Univ., JAPAN

Liborary, Kyoto Univ., JAPAN

Prot, N. Inown, Untr. of Tokyo, LAPAN

Secrotary, Pleama Section, Eloctrowchnical Lab., JAPAN

S. Mori, Technical Advisor, MAEF, LAPAN

Dr. O. Nund, Kumemolo inat. of Tectnology, JAPAN

J. Hyeon-Sook, Korea Atomic Eneroy Posearch Inst. KOAEA

D.I. Chat, The Korea Adv, Inst. of Sd. Tech., KOREA

Prof. B.S. Uloy, Univ, of Weikab, NEW ZEALAND

Inst of Phyaica, Chinese Acad Sad PEOPLE'S REP. OF CHINA

Librery, Inat. of Plaem Phyelas, PEOPLE'S AEP, OF CHINA

Tainghua Univ. Library, PEOPLE'S REPUBLIC OF CHINA

Z. L, S.W. Inst Phyeica, PEOPLE'S REPUBUC OF CHINA

Prof. J.A.C. Cabra, Imetituto SUperior Tecnico, PORTUGAL

Dr. O. Potrus, ALI CUZA UntV., ROMANIA

Dr. J. do Vilibers, Fucion Studies, AEC, S. AFAICA

Prot. M.A. Hewberg, Univ. of Natal, S. AFRICA

Prot. D.E. Km, Pohang Inat. of Sa. \& Toch., SO. KOREA

Prot. C.I.E.M.A.T, Fusion Division LJbrary, SPAIN

Dr. L Siontio, Univ. of UMEA, SWEDEN

Librery, Royd inat. of Tecmnology, SWEDEN

Prot. H. Whatmeon, Chaimers Univ, of Tech., SWEDEN

Centro Phys. Des Plasmas, Ecole Potytech, SWITZERLAND

Bibliotheek, Inat. Voor Plusma-Fysica, THE NETHERLANDS

Asat. Prot. Dr. S. Coldr, Midde East Tech. Univ., TURKEY

Dr. V.A. Gukhiwh,Sd. Ros. Inst. Electrophys. I Apparatus, USSR

Dr. D.D. Ayubr, Siborian Brench of Acadomy of Sal., USSA

Dr. G.A. Elleoon, I.V. Kurchator Inst., USSA

Lbrerien, The Ukr.SSR Academy of Scionces, USSR

Dr. LM. Kowrizinylth, Inst. of Genered Physica, USSP

Komtorechungeandege GmbH, Zentrabibliothek, W. GERMANY

Bibliothok, Inat. For Plasmatorechung, W. GERMANY

Prof. K. Sctinder, Ruhr-University Bochum, W. GERMANY

Dr. F. Weoner, (ASOEX), Max-Planck-Institut, W. GERMANY

Librerian, Max-Plenck-Institur, W. GERMANY

Prol. R.K. Janow, Inst. of Physics, YUGOSLAVIA 

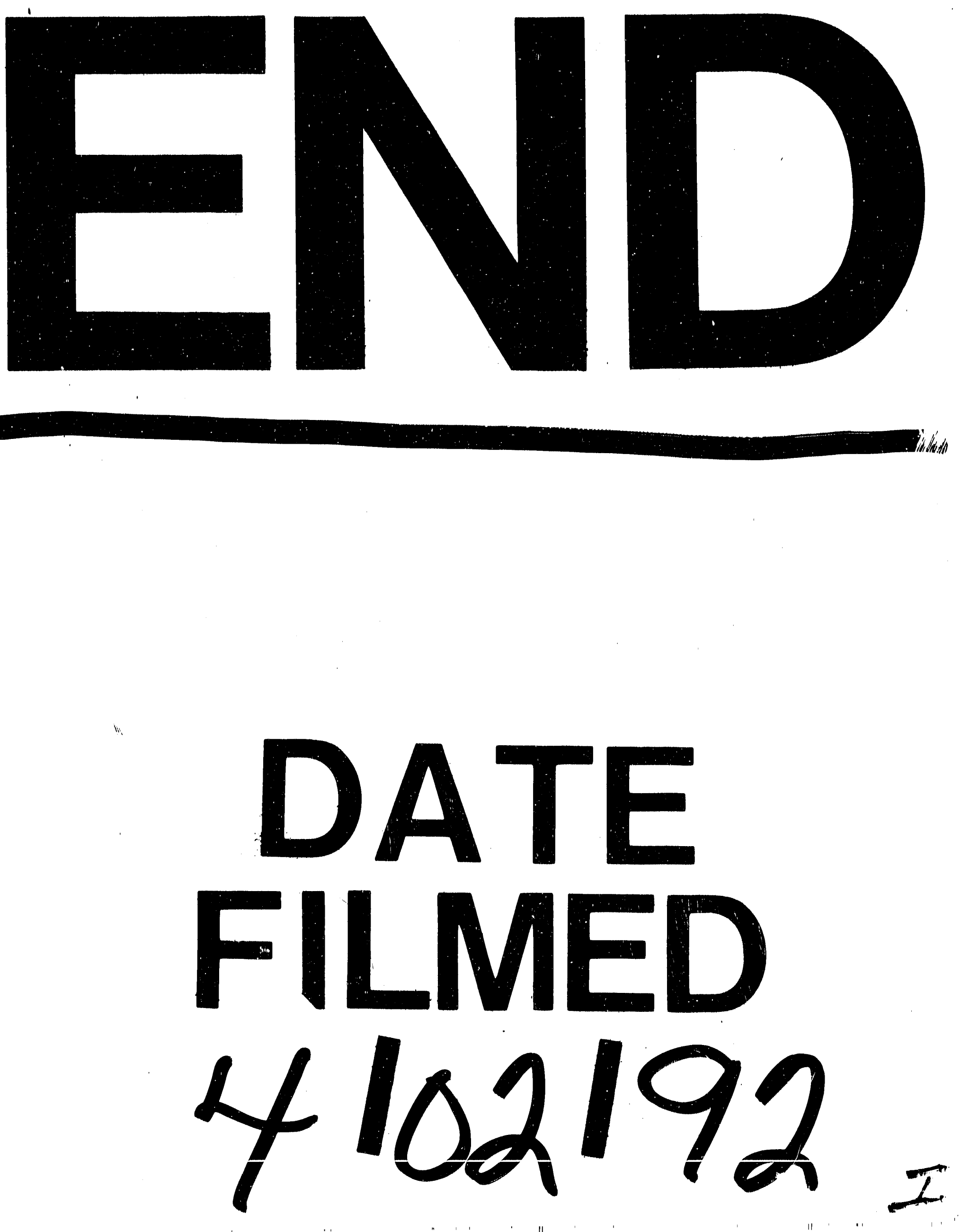
